\title{
Instrumentation for Millimeter-wave Magnetoelectrodynamic Investigations of Low-Dimensional Conductors and Superconductors
}

\author{
Monty Mola and Stephen Hill ${ }^{\dagger}$ \\ Department of Physics, Montana State University, Bozeman, MT 59717 \\ Philippe Goy \\ AB Millimètre, 52 Rue Lhomond, 75005 Paris, France
}

Michel Gross ${ }^{\S}$

Laboratoire KASTLER-BROSSEL, C.N.R.S. UMR 8552, and Université Pierre-et-Marie Curie, Département de Physique de l'Ecole normale supérieure, 24 Rue Lhomond, 75231 Paris, Cedex 05, France

(March 10, 2018)

\begin{abstract}
We describe instrumentation for conducting high sensitivity millimeter-wave cavity perturbation measurements over a broad frequency range $(40-200 \mathrm{GHz})$ and in the presence of strong magnetic fields (up to 33 tesla). A Millimeter-wave Vector Network Analyzer (MVNA) acts as a continuously tunable microwave source and phase sensitive detector $(8-350 \mathrm{GHz})$, enabling simultaneous measurements of the complex cavity parameters (resonance frequency and $Q$-value) at a rapid repetition rate $(\sim 10 \mathrm{kHz})$. We discuss the principal of operation of the MVNA and the construction of a probe for coupling the MVNA to various cylindrical resonator configurations which can easily be inserted into a high field magnet cryostat. We also present several experimental results which demonstrate the potential of the instrument for studies of low-dimensional conducting systems.
\end{abstract}

\section{Introduction}

Anisotropy, or low-dimensionality, plays a fundamental role in many areas of contemporary condensed matter physics, both pure and applied. For example, much of the technology which has revolutionized the microelectronics industry in recent years has resulted from the development of low-dimensional semiconductor devices. More recently, research into bulk layered materials such as the transition metal oxjdes, 1 organic conductors 3 semiconductor superlattices 3 magnetic nanostructures, $\mathrm{etc}$., has resulted in the discovery of a range of new physical phenomena, e.g. high temperature superconductivity, colossal magnetoresistance, 6 and a novel form of the quantum Hall effect 0 Many of these discoveries challenge our basic understanding of condensed matter physics while, at the same time, they hold the key to future technologies.

The use of microwave techniques to probe the electrodynamic properties of metals is not new. However, due to recent interest in a range of novel conducting systems in which the characteristic energy scales (e.g. electronic bandwidths, or energy gaps) coincide with the millimeter and sub-millimeter-wave spectral ranges, this field is undergoing a renaissance. Frequency dependent transport measurements in the presence of (strong) magnetic fields - the topic we call magnetoelectrodynamics, in analogy of magnetooptics - are expected to yield significant new insights into the physics of low-dimensional conductors, just as conventional magnetooptics and magnetotransport measurements were essential decades ago in leading to our present understanding of simple metals.

To date, few experiments on highly conducting materials have combined high magnetic fields and millimeter- wave spectroscopy 6 The reasons for this can be attributed to a range of factors. First, there are few commercially available sources and detectors working in this frequency range and those that can be used tend be highly priced, cumbersome to use, and possess either low power or an unstable output. Second, spectroscopy in this frequency range is complex, particularly in the case of metals: high sample reflectivity requires extreme sensitivity in order to detect small changes in conductivity; and the radiation wavelengths are typically larger than available samples, thus rendering conventional reflection spectroscopy useless. The inclusion of a magnetic field complicates these techniques considerably, since good optical coupling between the spectrometer (source and detector) and the sample under investigation is essential, and this is obviously difficult to achieve through the small bore (comparable to $\lambda$ ) of a large magnet.

In this paper we describe instrumentation which has been developed at Montana State University (MSU) for carrying out sensitive spectroscopy of low-dimensional conductors in the frequency range between 40 and 200 $\mathrm{GHz}$, and in magnetic fields up to 33 tesla which are available at the National High Magnetic Field Laboratory (NHMFL) in Tallahassee, FL. In order to a) maximize sensitivity, and b) achieve the required control over the electromagnetic field distribution in the vicinity of the sample, we utilize apgnclosed cavity perturbation technique (section II-B). age and outstanding dynamic range (signal-to-noise) are achieved using Millimeter-wave Vector Network Analyzer (MVNA) 10, 11, 12 as a source and detector (see section II-A). The resulting instrumentation offers unique experimental possibilities in the area of metals physics in 
high magnetic fields.

The paper is organized as follows: section II contains a detailed technical description of the system; in section III, we discuss the performance of the instrument; and we conclude with a summary of the paper in section IV.

\section{TECHNICAL DESCRIPTION}

\section{A. The MVNA}

As a source and detector, we employ Millimeterwave Vector Network Analyzer (MVNA) 10,11, 12 to monitor the phase and amplitude of millimeter-wave radiation transmitted through a resonant cavity containing the sample under investigation. The MVNA 8-350, which employs purely solid-state electronics, allows measurements over an extended frequency range $(8-350 \mathrm{GHz}) 13$ through harmonic multiplication of a sweepable centimeter source, $S_{1}$, which provides a nominally flat output power in the range $F_{1}=8-18 \mathrm{GHz}$. The mm-wave signal used in our experiments is extracted from a Shottky diode (harmonic generator - HG) which has been optimized to produce the desired harmonic $N$ of the sweepable $\mathrm{cm}$ source, i.e. $F_{m m}=N \times F_{1}$. Detection is then achieved by mixing the mm-wave signal $\left(F_{m m}\right)$ with the signal from a second cm source, $S_{2}$, at a second Schottky diode (harmonic mixer - HM). The beat frequency, $F_{\text {beat }}$, which preserves the phase and amplitude information of the mm-wave signal relative to the local oscillator $S_{2}$, is then sent to a heterodyne vector receiver (VR).

The low noise floor of the MVNA is achieved by defining the frequency difference between the two cm sources, $S_{1}$ and $S_{2}$, using a main oscillator. If one then uses the same harmonic rank on the source side (HG), and on the detection side (HM), the phase noise associated with the $\mathrm{cm}$ sources cancels in the beat signal $\left(F_{\text {beat }}\right)$ which is sent to the VR. Thus, the phase reference of the VR can be taken directly from the main oscillator.14

With the noise characteristics of the analyzer optimized, its dynamic range is limited only by the harmonic conversion efficiency of the Schottky diodes. At MSU, three pairs of Schottky diodes are used, operating in the $\mathrm{V}(\sim 45-70 \mathrm{GHz}, N=3$ and 4$), \mathrm{W}(70-110 \mathrm{GHz}$, $N=5$ and 6$)$ and D-bands $(110->200 \mathrm{GHz}, N=7$ to 12). The $\mathrm{V}$-band diodes are nominally flat broadband, while the $\mathrm{W}$ and $\mathrm{D}$ band diodes are mechanically tunable and require optimization each time the source frequency $\left(F_{1}\right)$ is changed. Operating in this mode, it is possible to perform bench-top tests up to $350 \mathrm{GHz}$. Table I lists the optimum dymamic ranges achieved at MSU (MVNA $8-350-1-2) 15$ in each frequency band, and for each harmonic, up to $200 \mathrm{GHz}(N=12)$. Similar analyzers are in use at seyeral high magnetic field laboratories around the world:16 in particular, the national magnet labs in the US and The Netherlands have MVNA 8-350 analyzers (with ESA options) 13 which are available to external users. The MVNA at the NHMFL, which has been used for some of the studies discussed in this paper, additionally operates in the Q $(30-50 \mathrm{GHz}, N=3), \mathrm{K}(16-32$
$\mathrm{GHz}, N=2)$ and $\mathrm{X}(8-18 \mathrm{GHz}, N=1)$ bands.

Although the $\mathrm{cm}$ sources $\left(S_{1}\right.$ and $\left.S_{2}\right)$ are phase (frequency) locked to each other, their absolute frequencies must be stabilized also. The frequency precision and stability provided by the MVNA 8-350 is not adequate for narrow-band cavity perturbation measurements when the bandwidth of the cavity is less than about 100 $\mathrm{MHz}$. For this reason, it is common to phase-lock one of the sources (i.e. both) to a quartz standard. At MSU and at the NHMFL, EIP 575 source-locking frequency counters 17 are used, which provide both the stability and precision necessary for the measurements described in this paper. One other mode of operation involves phase locking the source $\left(S_{1}\right)$ directly to the high $-Q$ cavity resonator used for the experiment. The counter is also useful in this case for recording changes in frequency resulting from any changes in dispersion within the cavity. Comparisons between the two frequency locking techniques are discussed in section III-B.

The dynamic ranges listed in Table I represent ideal values for the MVNA 8-350 assuming there are no insertion losses between HG and HM. Due to the considerable size of a typical magnet cryostat and, in particular, the dimension separating the magnetic field center and the top of the cryostat ( $\sim 1.5 \mathrm{~m}$ in our case), a considerable insertion loss is unavoidable. Furthermore, it is essential to keep a reasonable distance $(\sim 2 \mathrm{~m})$ between the MVNA and the magnet, since the cm sources (YIG oscillators) are sensitive to stray magnetic fields of more than a few Gauss. The Schottky diodes (HG and HM), on the other hand, are not field sensitive and may be placed closer to the experiment. Thus, the cm and beat frequencies are propagated between the MVNA and Schottky diodes through flexible coaxial cables, which introduce a combined insertion loss of 4 to $5 \mathrm{~dB}$. Meanwhile, the mm-wave signal is propagated from the HG to the cavity within the magnet cryostat, and back to the HM, using a pair of rigid waveguides. These waveguides account for a major part of the insertion loss of the system; minimizing these losses is the subject of section II-B.1. A schematic of this arrangement is shown in Fig. 1.

In addition to its use as a source and detector for solid state spectroscopy, the network analysis capability of the MVNA is crucial during the developmental stages of a measurement system. The frequency sweeping capability can be used to determine the precise location of impedance mis-matches and/or microwave leaks from the system, and for the characterization and optimization of different resonator designs.

\section{B. The sample probe}

As outlined in the introduction, our aim is to be able to conduct sensitive cavity perturbation measurements, at low temperatures, high magnetic fields and over a broad frequency range. In this section, we describe an experimental scheme - the "sample probe" - for coupling the MVNA to various enclosed cylindrical resonator configurations which can easily be inserted into a high field 
magnet cryostat. The "sample probe" refers to the passive microwave hardware which has been developed at MSU and is compatible with the MSU and NHMFL magnet systems. It consists of the following components: two long waveguides for propagating mm-waves from the HG, into the magnet cryostat (incidence waveguide), and back to the HM (transmission waveguide); a demountable cavity, of which we have developed two standard types; a coupling between the cavity and the incidence and transmission waveguides; a vacuum tube to isolate the probe from the surrounding liquid cryogens; and electronics for controlling the sample temperature, and for magnetic field modulation. Fig. 1 shows a schematic of the probe situated within the superconducting magnet cryostat at MSU, and Fig. 2 shows an equivalent LRC circuit representation of the key microwave components; we defer explanation of these figures until following sections, where various aspects of the probe design are discussed in detail.

From the outset, we established two key experimental objectives: i) it should be possible to make measurements at several well separated frequencies without the need to interfere with, or warm up, the sample probe; and ii) the sensitivity, dynamic range and mechanical stability of the measurement system should be optimized as far as possible. The flexibility in choice of frequency is important for experiments at the NHMFL, where magnet time is often limited. There are also other advantages to this, e.g. by not having to interfere with the sample, it is possible to ensure that it sits in an identical electromagnetic field distribution for a given series of cavity modes. The second goal is fairly self explanatory, nevertheless, high sensitivity comes at the expense of some flexibility. We have, therefore, opted for a rigid construction, i.e. no insitu rotation of either the cavity or the sample within the cavity is possible. Our experience has shown that such mechanisms result in radiation leakage from the cavity and diminished sensitivity.

\section{Reducing losses in the waveguides}

The success of our recent measurements owes as much to reductions in the insertion losses associated with our sample probe, as it does to the optimization of the cavity designs. In this section, we discuss the technical details of how to propagate $\mathrm{mm}$-wave radiation from the Schottky diodes into a low temperature cryostat (pumped ${ }^{4} \mathrm{He}$ ) within the bore of a high field magnet system where the resonant cavity containing the sample is situated. As illustrated in Fig. 1, the MSU setup utilizes a separate inner cryostat to control the temperature of the experiment. This cryostat, which sits within the bore of the superconducting magnet, draws liquid helium from the main reservoir into its tail, which is thermally isolated from the main helium reservoir, thus allowing good control over the sample temperature.

The microwave probe is based on $\mathrm{V}$-band rectangular waveguides which are terminated at their source/detector ends with UG385/U flanges for convenient connection to the Schottky diodes 18 V-band waveguide cuts off below $\sim 45 \mathrm{GHz}$, which is sufficiently low for most of the intended applications, and is also the lowest frequency band currently available at MSU. Furthermore, the narrow bores of the magnets at the NHMFL19 essentially rule out the possibility of using waveguides below Q-band (cut-off at $33 \mathrm{GHz}$ ) for transmission measurements.

Commonly used low loss, high conductivity, waveguide materials such as copper or silver are not ideal for our purposes. Unacceptable heat flow down the waveguides and into the cryostat leads to excessive liquid helium boil-off and limits the ability to cool the cavity/sample to pumped ${ }^{4} \mathrm{He}$ temperatures $(\sim 1.5 \mathrm{~K})$. For cryogenic purposes, Stainless Steel (SS) waveguide offers an attractive alternative.18, 0 it has both a low thermal conductivity,21 and it is possible to use thinner walled waveguide material.22 Unfortunately the microwave losses in SS waveguides are severe, as illustrated in Fig. 3, which shows a comparison between the insertion losses of SS and Ag, as measured using the frequency sweeping capability of the MVNA; note that, in this range, the loss in $\mathrm{SS}$ exceeds $10 \mathrm{~dB} / \mathrm{m}$, whereas the loss in $\mathrm{Ag}$ is less than $2 \mathrm{~dB} / \mathrm{m} 23$ The solution, therefore, is to construct composite waveguides from highly conducting waveguide sections isolated by SS sections. Although this necessitates several joints in the waveguide assembly, we have found that these do not significantly impair the functionality of the waveguide.

Placement of the SS waveguide sections is crucial to the cryogenic performance of the system, as illustrated in Fig. 1. The largest temperature gradient occurs in the upper part of the magnet dewar where the radiation baffles are situated. It is in this region that we first use SS. We again use short SS sections at the lower end of the probe in order to thermally isolate the cavity/sample from the long $\mathrm{Cu} / \mathrm{Ag} 2$ sections which run from just below the baffles, through the liquid ${ }^{4} \mathrm{He}$ reservoir, and into the bore of the magnet. The cavity and sample are, therefore, more-or-less isolated from the main $4.2 \mathrm{~K}$ helium reservoir, enabling control over the temperature of the cavity/sample from about $1.35 \mathrm{~K}$, up to 40 or $50 \mathrm{~K}$ (see section II-B.4). The lower SS waveguide sections have been gold plated 25 for reasons which are discussed in the following section.

One unforeseen benefit of the composite waveguide construction, which greatly improves the mechanical stability of the probe, is its insensitivity to the liquid helium level in the magnet cryostat. We assume that the $\mathrm{Cu} / \mathrm{Ag}$ section maintains a fairly uniform temperature $(\sim 4.2 \mathrm{~K})$ over its entire length due to its high thermal conductivity. Thus, the temperature gradient in the SS section does not depend on the liquid helium level. In contrast, the thermal gradient in a single SS section immersed in liquid helium would depend on the level of the liquid, causing the waveguide to expand slightly over time, thereby affecting the microwave phase stability of the system. 
The $\mathrm{SS}$ and $\mathrm{Cu} / \mathrm{Ag}$ rectangular waveguide sections, which have different outer dimensions, 22 are coupled together using specially machined clamps which screw tightly around the waveguides.20 The joints are staggered so as to minimize cross talk between the waveguides due to any microwave leakage from the joints (see following section). Thus, although both waveguides pass through each clamp, only one of the waveguides is joined at each clamp. This construction is extremely rugged, yet it easily permits modifications in the overall probe length, and in the placement of the SS sections. Therefore, the same waveguide assembly can easily be re-configured for use at other magnet facilities such as the NHMFL.

Finally, the entire sample probe fits tightly inside a $19.05 \mathrm{~mm}$ OD $(0.4 \mathrm{~mm}$ wall) vacuum jacket. The integrity of the vacuum is maintained across the waveguides at their room temperature ends using mylar windows clamped between standard flanges (UG385/U) which have been modified to hold rubber 'O'-rings. Again, we offset the vacuum seals on the incident and transmission waveguides, and wrap them in steel wool to minimize cross-talk. A hermetically sealed connector provides 19 electrical feedthroughs for thermometry, field modulation coils, and for powering a heater.

\section{Minimizing undesirable instrumental effects}

The basic principal behind the cavity perturbation technique is relatively simple, and involves measuring changes in the complex cavity parameters (i.e. resonapce frequency $f_{o}$ and $Q$-value) upon insertion of a sample 8 , Relating these changes to the complex electrodynamic properties of the sample can be a formidabtact, and we refer the reader to a series of three articles 27.28.29 dealing with this analysis for precisely the types of materials which we set out to study in high magnetic fields, i.e. highly anisotropic crystalline conductors. However, in order to reliably apply such an analysis in our case, it is essential that we first consider how each element in the sample probe affects our ability to extract the relevant information from the cavity mounted at the end of the probe. As we shall show, this is a formidable task in itself, which is complicated considerably by the restricted access into the bore of a typical high field magnet cryostat, and by the need for the probe to accommodate cavities resonating over a broad range of frequencies.

The task of optimizing the probe design is best tackled by considering an equivalent $\mathrm{AC}$ circuit (Fig. 2). Each component of the probe may be modeled as a self contained LRC circuit which is inductively coupled to the next. The Schottky diodes, HG and HM, attach to the upper ends of the incidence and transmission waveguides, which have impedances $\mathbf{Z}_{I}$ and $\mathbf{Z}_{T}$, respectively. The coupling between HG (HM) and the incidence (transmission) waveguide is modeled as a coupling mutual inductance $m_{H G}\left(m_{H M}\right)$. The cavity, which is mounted at the lower end of the two waveguides and has impedance $\mathbf{Z}_{C}$, is coupled to each of the waveguides through coupling mutual input $\left(m_{i n}\right)$ and output $\left(m_{\text {out }}\right)$ inductances. Ideally, the incidence and transmission waveguides should be coupled solely through the cavity. However, a leak signal between the incidence and transmission waveguides, in parallel with the cavity, is inevitable; this is modeled as a direct coupling mutual inductance, $m_{l}$, between the two waveguides.

It is changes in $\mathbf{Z}_{C}$, caused by the insertion of a sample into the cavity, which one would like to measure in a cavity perturbation experiment. Since the MVNA measures phase and amplitude, it is important to see how $\mathbf{Z}_{C}$ affects both of these parameters. Before, considering the probe as a whole, i.e. the entire circuit in Fig. 2, we examine the cavity by itself. Variations in the amplitude and phase of a wave transmitted through a cavity, as the frequency $(f)$ is swept across a resonance, are given by

$$
A^{2}(f)=\frac{1}{1+\left[2\left(f-f_{o}\right) / \Gamma\right]^{2}}
$$

and

$$
\phi(f)=-\arctan \frac{2\left(f-f_{o}\right)}{\Gamma},
$$

where $f_{o}$ is the center frequency and $\Gamma$ is the FWHM of the resonance; these expressions have been normalized so that $A=1$ and $\phi=0$ at resonance. As $f$ is swept from 0 to $\infty, A$ and $f$ sweep out a circle in the complex plane, where the real and imaginary amplitudes are given by $A_{1}(f)=A \cos \phi$ and $A_{2}(f)=A \sin \phi$ respectively, or

$$
A_{1}(f)=\frac{1}{1+\left[2\left(f-f_{o}\right) / \Gamma\right]^{2}}
$$

and

$$
A_{2}(f)=\frac{2\left(f-f_{o}\right) / \Gamma}{1+\left[2\left(f-f_{o}\right) / \Gamma\right]^{2}},
$$

the more familiar expressions for a Lorentzian. Such a circle is shown in Fig $4 \mathrm{a}$, for a resonance at $60 \mathrm{GHz}$, with a $Q$ of 5000. Each point in the figure corresponds to a different frequency, and the frequency interval between each point is $800 \mathrm{kHz}$. Points closest to the origin correspond to $f \ll 60 \mathrm{GHz}$ and $f \gg 60 \mathrm{GHz}$. The resonance frequency $\left(f_{o}\right)$ lies along the real axis $(\phi=0)$ where the spacing between points is greatest, i.e. when the phase rotates most rapidly with frequency. Clockwise rotation around the circle represents increasing frequency. The corresponding changes in $A(f) \& \phi(f)$ and $A_{1}(f) \& A_{2}(f)$ are shown in Figs $4 \mathrm{~b}$ and c, respectively.

In a locked frequency experiment, changes in dispersion $\left(\operatorname{Im}\left\{\mathbf{Z}_{C}\right\}\right)$ cause the cavity to go off resonance, i.e. the point corresponding to $f_{o}$ in Fig. 4 a will move away from the real axis. Provided that this effect is weak (a perturbation), the dominant result is a change in the phase of the signal transmitted through the cavity, and no appreciable change in amplitude. Conversely, changes in dissipation $\left(\operatorname{Re}\left\{\mathbf{Z}_{C}\right\}\right)$ within the cavity will result in 
a reduction in the amplitude of the signal transmitted through the cavity and, hence, a reduction in the diameter of the circle in Fig. 4a. Dissipation alone does not move $f_{o}$ away from the real axis and, therefore, does not affect the phase of the wave. However, dispersion can affect both the amplitude and the phase of the wave if $f_{o}$ moves appreciably off the real axis. For this reason, it is often desirable to conduct a locked phase experiment, which completely decouples these two effects. With a phase lock, the cavity stays on resonance (on the real axis in Fig. 4a), and dispersion affects the resonance frequency only, which we can measure with a frequency counter. Meanwhile, changes in dissipation again affect the amplitude of the transmitted signal only.

Unfortunately, each of the additional circuit elements required to link the MVNA to the cavity, and/or improper coupling between these components, has the potential to seriously distort the simple relationships between dissipation, dispersion, amplitude and phase discussed above. Ideally, the sample probe should be passive, low loss, insensitive to temperature and magnetic field and, with the exception of the cavity, should have a flat broad-band frequency response. In practice, this is never actually possible to achieve. Nevertheless, by conducting a thorough characterization and optimization of each element in the microwave circuit (Fig. 2), it is possible to minimize these instrumental effects to negligible levels. The MVNA performs a pivotal role in this hardware developmental process. The following paragraphs discuss various undesirable instrumental characteristics, their potential effect on a measurement, and the steps we have taken to eliminate these sources of error.

A leak wave bypassing the cavity directly through to the transmission waveguide has two adverse effects. First, it diminishes the useful dynamic range - ideally $100 \%$ of the signal reaching the detector should pass through the cavity. Second, if the leak amplitude is comparable to the amplitude of the signal passing through the cavity, the resonance may become severely distorted, making it extremely difficult to distinguish between dissipative and dispersive effects within the cavity. As illustrated in Fig. 5, a leak wave adds a complex vector to the signal transmitted through the cavity. By minimizing the leak, one can control its amplitude. However, it is not possible to control the phase of the leak wave. Consequently, big leaks lead to an arbitrary vector translation of the circle in Fig. 4a; this is a pure translation, i.e. each point on the circle is translated by the same vector. Hence, the line joining the resonance frequency, $f_{o}$, and the $f=0 \& \infty$ points, remains parallel to the real axis. As a result, the transmitted amplitude on resonance is not necessarily the maximum amplitude; indeed, it can take on any value from zero to one plus the leak amplitude. This is illustrated in Fig. 5 for an arbitrary translation of the circle in Fig. 4a, together with the corresponding variations in phase and amplitude, plotted versus frequency. It is apparent from this figure that the phase of the leak signal is entirely responsible for the way in which dissipation and dispersion affect the phase and amplitude of the signal transmitted through the cavity. Thus, an appreciable leak signal is intolerable, and we have taken every step to reduce the leak in our probe to at least $20 \mathrm{~dB}$ below the typical signal transmitted through the cavity on resonance, as discussed above.

Standing waves in the waveguides are unavoidable and, without proper attention, can cause considerable problems, especially when operating in the phase-locked mode in which the incident mm-wave frequency is locked to the cavity resonance frequency. Changes in this frequency will result in changes in the phase and amplitude of the mm-wave signal incident upon the cavity. Thus, it becomes impossible to distinguish between the intrinsic cavity response and spurious effects due to the standing waves. Furthermore, the phase is no longer truly locked to the cavity resonance under these circumstances, but rather to the coupled response of the entire circuit in Fig. 2. Standing waves should not be ignored altogether in the frequency locked mode either, particularly when the cavity is well coupled to the waveguides. Under these circumstances, changes in $\mathbf{Z}_{C}$ influence the impedance matching between the cavity and the waveguides (i.e. $m_{\text {in }}$ and $\left.m_{\text {out }}\right)$ and, therefore, affect the standing waves.

There is little to be gained from trying to eliminate the standing waves completely. This would require precise impedance matching of each component in Fig. 2, which is only possible to achieve over a narrow frequency range and would, therefore, defeat the purpose of the probe, which is intended to work over a fairly broad frequency range. Instead, we concentrate on minimizing the influence of the standing waves on a measurement at any given frequency. This is achieved by reducing the frequency bandwidth of the measurement to well below the periodicity of the standing wave pattern, i.e. so that the response of the waveguides is essentially flat over the relevant frequency interval. In this way, a range of cavities or cavity modes may be utilized, covering an extremely broad frequency range in comparison to the standing wave periodicity. Meanwhile, each cavity mode samples only a minute portion of the waveguide spectrum, over which its response is essentially flat.

The fastest standing wave period is governed by the longest dimension of the sample probe, which is $3 \mathrm{~m}$ in our case (HG to HM, via the cavity). This gives rise to a standing wave periodicity of about $100 \mathrm{MHz}$ which, in turn, requires cavity filling factors of less than $10^{-4}$, so that the frequency shift in any given measurement never exceeds about $10^{-4}$ of the measurement frequency, i.e. $\Delta f_{o}<10 \mathrm{MHz}$ for $f<100 \mathrm{GHz}$. Cavity filling factors of between $10^{-5}$ and $10^{-4}$ are typical for the types of samples we study, so standing wave problems do not force this restriction upon us. Nevertheless, to compensate for the small filling factors, it is essential to have cavity $Q$-factors on the order of $10^{4}$. In order to attain such high $Q$-values, the cavity must necessarily be 
coupled weakly to the waveguides, which further reduces problems associated with standing waves.

Phase instability, and/or a strong frequency dependence of the phase reaching the VR, can lead to a variety of problems. Although the internal sources within the MVNA are phase locked, additional phase jitter can arise in the mm-wave signal due to mechanical and/or thermal instabilities in the sample probe. Mechanical vibrations do not pose any problems, either at MSU or at the NHMFL. However, thermal stability is essential for achieving the best results. Due to extremely high cavity $Q$-values, minor temperature fluctuations can lead to significant phase instabilities, particularly when studying samples with a strongly temperature dependent electrodynamic response. For this reason, active temperature control is necessary (see section II-B.4). Long term thermal stability of the entire probe, including the $1.5 \mathrm{~m}$ waveguide sections, is also desirable and is best achieved using the composite waveguides described in the previous section.

A strong frequency dependence of the phase reaching the VR occurs when the distances between the sources $S_{1}$ and $S_{2}$ and the HM are very different. The signal originating at $S_{1}$ has to travel the extra $2 \times 1.5 \mathrm{~m}$ into, and out of, the magnet cryostat. For this reason, it can be beneficial to compensate for the extra distance between $S_{1}$ and the HM by adding $3 \mathrm{~m}$ of coaxial cable 30 between $S_{2}$ and the HM, especially when performing phase-locked or frequency swept measurements, even though this introduces an extra $2-3 \mathrm{~dB}$ insertion loss.

Spurious magnetic resonances caused by paramagnetic contamination of either the cavity, or the waveguides close to the magnetic field center, will give rise to both sharp and broad instrumental features (Electron Paramagnetic Resonances - EPR) in the transmission versus magnetic field response of the system. These spurious resonances can be hard to distinguish from the genuine response of the sample within the cavity, and should be eliminated so that the magnetic field response of the unloaded probe (i.e. with no sample) is as flat as possible. The $\mathrm{SS}$ waveguide sections cause the most severe problems, which we suspect is due to the presence of small traces of $\mathrm{Fe}^{3+}$ (rust) at the surface of the metal. For this reason, we have gold plated the SS sections which couple directly to the cavity. This not only reduces the insertion loss due to these sections, but also completely eliminates a broad instrumental resonance, leaving an extremely flat response $(\Delta A<0.05 \mathrm{~dB})$, as shown in Fig. 6. A number of other measures have been taken to avoid contaminating the lower end of the probe (i.e. close to the field center) with paramagnetic impurities. These include clamping all components together rather than using adhesives or solders, and avoiding the use of cutting oils when machining the resonators. We have also gold plated some of the cavities to prevent tarnishing. Nevertheless, electrolytic OFHC Cu cavities work extremely well without plating (see Fig. 6).

\section{The cavities}

To date, our efforts have focussed on the use of enclosed cylindrical resonators for cavity perturbation measurements. There are several compelling reasons to do so. To begin with, the simple design concept we have developed, in which the cavities are assembled from relatively few easily machinable components (see Figs. 7 and 8), enables us to fabricate a range of cavities and, therefore, switch frequency range/coverage with relative ease. 31 More importantly, relative to rectangular cavities, it is straightforward to achieve extremely high $Q$-values $\left(>10^{4}\right)$ for the TE01 $n(n=1,2,3 \ldots)$ modes of cylindrical resonators. The reason for this has to do with the fact that no AC currents flow between the end and side walls of a cylindrical cavity excited in its TE01 $n$ mode. Consequently, joints at these ideal locations (see Figs 7 and 8) do not diminish the cavity $Q$-factor. These modes also possess electromagnetic field geometries which are highly desirable for the measurements described in this paper.

\subsection{Axial cavities}

For the most part, we use axial cavities of the type shown in Fig. 7, i.e. ones in which the cavity axis is coincident with the axis of the superconducting solenoid. This is by far the most versatile design, though it does have some limitations, especially when it is necessary to tilt the sample with respect to the applied DC magnetic field. The cavity is constructed from OFHC electrolytic copper in three pieces - a blank end plate, a coupling plate and a cylindrical barrel. This assembly bolts onto the under side of a copper housing which clamps around the incidence and transmission waveguides. These bolts also provide the pressure for reproducibly clamping the cavity assembly tightly together; no adhesives or solders are used. The cavity may be disconnected from the waveguides in a matter of seconds for easy sample insertion/removal. The housing which clamps around the waveguides is also easily interchanged with a separate housing for a transverse cylindrical cavity (see below).

Coupling between the waveguides and the cavity is achieved by means of small circular apertures in the thin coupling plates. Since these plates terminate the waveguides and the cavity, there are no transverse microwave electric $(\tilde{\mathbf{E}}-)$ fields at the locations of the apertures. Thus, it is the microwave magnetic $(\tilde{\mathbf{H}}-)$ fields in the waveguides and the cavity which should be matched. For the TE01n modes, the $\tilde{\mathbf{H}}$-fields flow radially at the cavity ends and, therefore, the $\tilde{\mathbf{H}}$-fields in the waveguides should do so also. For this reason, the incoming waveguides are oriented with heir shortest edges closest together (see Figs $1,7$ and 8$) .32$

We can control the degree of coupling between the waveguides and the cavity $\left(m_{\text {in }}\right.$ and $\left.m_{\text {out }}\right)$ by means of the dimensions of the coupling apertures (diameter and thickness). There is an important trade off here between i) strong coupling (large apertures), which ensures good power throughput from the source to the detector 
and, hence, a large dynamic range, and ii) weak coupling (small apertures), which limits radiation losses from the cavity, resulting in higher cavity $Q$-values and increased sensitivity, at the expense of some dynamic range. We have found empirically that the optimum coupling apertures should be small for our setup, with diameter $\sim \lambda / 4$. It is also necessary for the coupling plate to be very thin $(\sim \lambda / 20)$, since the signal is obviously attenuated as it passes through the apertures, which are way below cutoff. The relatively weak coupling to the cavity makes it all the more important to reduce any/all other losses in the sample probe and, hence, preserve a reasonable dynamic range, i.e. without taking steps to reduce losses in the waveguides (see section II-B.1), we would be forced to increase the coupling, resulting in reduced sensitivity.

The resonance frequency of a particular cavity is determined by the length and the inner diameter of the cylindrical barrel (see Fig. 7). Because of the simplicity of machining this section, it is no great task to construct a large number of cavities, providing many TE01 $n$ modes covering a wide range of frequencies. However, because of the wavelength dependence of the coupling between the waveguides and the cavity, it is also necessary to construct several different coupling plates - roughly one for each frequency band, i.e. $\mathrm{V}-, \mathrm{W}-, \mathrm{D}_{-}$, etc.. Because the inner diameters of the cavities generally get smaller at higher frequencies, the positioning of the apertures is also critical. In all cases, the input and output coupling apertures are located diametrically opposite each other, and at the same radial distance from the axis of the cavity. In order to minimize the number of these coupling plates, we have limited the cavity diameters to four standard sizes. For V-band (cavity dia. $=9.52 \mathrm{~mm}$ ), the coupling apertures are located at the optimum positions, both with respect to the cavity and the waveguides, i.e. in the middle of the waveguides and half way between the cavity axis and its perimeter. For the higher frequency bands, a compromise between these positions is made.

A clear gap of $0.51 \mathrm{~mm}$ is maintained between the incidence and transmission waveguides, which terminate flush with the under side of the cavity housing. The coupling plate is machined with a $0.51 \mathrm{~mm}$ wide, $0.75 \mathrm{~mm}$ high, ridge running perpendicular to the line joining the coupling apertures, and intersecting the mid point between them (see Fig. 7). This ridge locates inside a matching groove on the under side of the cavity housing and, therefore, in between the incidence and transmission waveguides, as shown in Fig. 7. We have found that it is this joint between the cavity and the waveguides which is most susceptible to microwave leaks, and that the ridge in the coupling plate dramatically reduces the leak amplitude. The leak may be reduced further still by applying a small amount of Indium into the groove before assembling the cavity.

For a perfectly cylindrical cavity, the TE01n modes are degenerate with the TM11n modes. It is a trivial task to lift this degeneracy without diminishing the $Q$-values of the TE01n modes. We achieve this by drilling a small indent in the center of the blank end plate where essentially no currents flow for the TE01n modes; this hole may also be used to hold a quartz pillar for mounting a sample on axis above the cavity end plate (see Fig. 9). Consequently, the TM modes are shifted to lower frequencies by up to $200 \mathrm{MHz}$, they are also weaker and have lower $Q$-values than the TE modes.

\subsection{Transverse cavities}

Recently, we have developed a transverse cavity optimized to work in V-band (shown in Fig. 8). Although the construction of this cavity is a little more complex than the axial cavities, it offers a major advantage for experiments in which it is necessary to tilt the sample relative to the applied DC magnetic field. The major difference between this cavity and the axial one is that the mm-waves are coupled through apertures drilled directly through the side wall of the cylindrical barrel, i.e. a separate coupling plate is not employed. This requires removing material from one side of the cylindrical barrel so that the side wall of the cavity is sufficiently thin in the vicinity of the coupling apertures. This flat surface also facilitates attachment to the underside of a specially designed cavity housing, which again clamps around the incidence and transmission waveguides. The cavity end plates simply bolt onto either end of the cavity barrel; one of these end plates has machined slots at the bolt circle radius so that it may be rotated about the cavity axis.

As with the axial cavity, we again implement a ridge/groove arrangement between the waveguides and the cavity, in order to minimize any microwave leakage at this position. The locations of the coupling apertures in the side wall of the cavity have been optimized for exciting the TE012 mode, i.e. at ${ }^{1 / 4} h$ and $3 /{ }_{4} h$ from the ends of the cavity, where $h$ is the cavity height. Nevertheless, these positions provide good coupling to the TE011 mode and, to a lesser extent, the TE013 mode.

\subsection{Sample positioning, and sample rotation}

Using one or other of the two cavity types, it is possible to subject a sample to virtually any combination of AC $\tilde{\mathbf{E}}$ - and $\tilde{\mathbf{H}}$-field polarizations, relative to the applied DC magnetic field $\left(\mathbf{B}_{\mathbf{o}}\right)$ orientation, e.g. at an $\tilde{\mathbf{H}}$ node with $\tilde{\mathbf{E}} / / \mathbf{B}_{\mathbf{o}}$, or at an $\tilde{\mathbf{E}}$ node with $\tilde{\mathbf{H}} \perp \mathbf{B}_{\mathbf{o}}$, etc. However, it turns out that for studies of highly anisotropic conductors, two convenient locations are sufficient for most experiments utilizing TE01n modes. 8.28 In the "end plate" configuration, the sample is placed on the blank cavity end plate, exactly half way between the cavity axis and its perimeter, as shown in Fig. 9a. In the "quartz pillar" configuration, the sample is mounted atop a thin quartz pillar (dia. $=0.71 \mathrm{~mm}$ ) on the axis of the cavity, as shown in Fig. 9b. In both of these configurations, the sample sits in an $\tilde{\mathbf{H}}$-field antinode for the TE011 mode, the polarization of which is radial for the end plate configuration 
and axial for the quartz pillar configuration (see Figs 9a and $b$ ).

For the end plate configuration, all TE01 $n$ modes have radial $\tilde{\mathbf{H}}$-field antinodes at the sample location. This is not the case for the quartz pillar configuration; for example, if the sample is mounted precisely mid-way between the cavity end plates, the even $n$ modes have both $\tilde{\mathbf{E}}$ and $\tilde{\mathbf{H}}$-field nodes at this location. Careful forethought as to the positioning of the sample can rectify this problem to a certain extent. However, whenever positioning the sample away from the mid-point ( $\tilde{\mathbf{H}}_{\max }$-point) of the cavity, sensitivity is compromised. Indeed, even the end plate position is appreciably less sensitive than the cavity mid-point 28

To understand how it is that the end plate and quartz pillar locations within a TE01n cavity can be sufficient for studying anisotropic systems, we consider the electrodynamics of a quasi-two-dimensional (Q2D) conductor. We assume a quasi-static approximation and consider the Faraday $\tilde{\mathbf{E}}_{F}$-field resulting from the time varying $\tilde{\mathbf{H}}$-field, i.e. an oscillatory $\tilde{\mathbf{E}}_{F}$-field which curls around the polarization of the $\tilde{\mathbf{H}}$-field. In an isotropic conductor, this Faraday field would induce circulating currents in a plane perpendicular to the $\tilde{\mathbf{H}}$-field. Indeed, this is approximately what happens if the $\tilde{\mathbf{H}}$-field is polarized perpendicular to the highly conducting planes of a Q2D conductor, as illustrated in Fig. 9c. However, because of the high in-plane conductivity, these induced currents are damped within the interior of the sample, i.e. currents only circulate within the skin layer at the edge of the sample. Consequently, the in-plane complex surface impedance, $\mathbf{Z}_{S}=\mathbf{R}_{S}+i \mathbf{X}_{S}$, governs the electrodynamic response of the sample in this situation, where $\mathbf{R}_{S}$ and $\mathbf{X}_{S}$ are the surface resistance and reactance respectively.272820 If, instead, the $\tilde{\mathbf{H}}$-field is polarized parallel to the highly conducting layers, $\tilde{\mathbf{E}}_{F}$ will induce both in-plane and inter-layer currents. As before, the in-plane currents will only flow at the edges of the sample, whereas the inter-layer currents flow throughout the bulk of the sample, due to the poor conductivity in this direction. Consequently, the complex inter-layer conductivity dominates the electrodynamic response of the sample under these conditions, as depicted in Fig. $9 \mathrm{~d} .27 .28 .29$

Of major interest for studies of low-dimensional conductors is the possibility to rotate the applied DC magnetic field, $\mathbf{B}_{\mathbf{o}}$, with respect to the sample. Since the cavities are rigidly connected to the sample probe, the orientation of $\mathbf{B}_{\mathbf{o}}$ cannot be adjusted relative to the cavity, i.e. $\mathbf{B}_{\mathbf{o}}$ is fixed parallel to the cavity axis for the axial cavity, and perpendicular to the axis for the transverse cavity. Thus, the axial cavity essentially limits investigation to only two orientations of $\mathbf{B}_{\mathbf{o}}$ relative to the sample, namely parallel or perpendicular to the Q2D layers, which we denote $\mathbf{B}_{/ /}$and $\mathbf{B}_{\perp}$ respectively. One could tilt the sample away from either of these geometries within the cavity. However, this would result in a misalignment of the $\tilde{\mathbf{H}}$-field polarization relative to the sample. This is the main reason for developing the transverse cavity. Because of the cylindrical symmetry of the TE01n modes, one can rotate the sample about the cavity axis by means of rotating the cavity end plate to which it is attached, without affecting the polarization of $\tilde{\mathbf{H}}$ relative to the sample; this works for both the end plate and quartz pillar configurations, as illustrated in Fig. 9e.

\section{Temperature and magnetic field control}

Being constructed from a block of high conductivity copper, the cavity makes an excellent heat reservoir for controlling the temperature of the sample, which is kept in good thermal contact with the cavity at all times, either by directly attaching it to the cavity end plate, or by mounting it on a quartz pillar; Silicone grease is used to hold the sample in place. A coil of high resistance wire is used as a heater. This coil is wound around a pillar which screws into the cavity housing and is, therefore, easily interchanged between the various cavity geometries (not shown in Figs 7 and 8). Low pressure helium gas is admitted into the $19.05 \mathrm{~mm}$ vacuum jacket for exchanging heat between the sample probe and the surrounding pumped liquid helium cryostat. Thus, the cavity and the sample can accurately and controllably be maintained at any temperature in the range from $1.35 \mathrm{~K}$ up to about $50 \mathrm{~K}$. The temperature is stabilized according to a calibrated Cernox resistance thermometer embedded in the walls of the copper cavity. Cernox thermometers have negligible magnetoresistance above $4.2 \mathrm{~K}$, and we can correct for a weak magnetoresistance below $4.2 \mathrm{~K}$.

Magnetic fields at MSU are generated using a standard commercial superconducting solenoid and power supply. This magnet routinely operates to $8 \mathrm{~T}$, and will go to $9 \mathrm{~T}$ at pumped liquid helium temperatures. At the NHMFL, the strongest magnetic fields are produced in axial Bittertype water cooled resistive magnets powered by $20 \mathrm{MW}$ supplies Presently, the highest available continuous field is $33 \mathrm{~T} .33$ Retails of the NHMFL magnets are published elsewhere. 34

\section{PERFORMANCE}

\section{A. Tests}

Fig. 10 shows a frequency sweep across the TE011 mode of an axial cavity (cavity $\mathrm{A}$, height $=6.7 \mathrm{~mm}$, dia. $=9.52 \mathrm{~mm}$, see Table II); the cavity is loaded with a sample of $\kappa$-(BEDT-TTF $)_{2} \mathrm{Cu}(\mathrm{SCN})_{2}$ (approx. dimensions $\left.0.5 \times 0.50 \times 0.2 \mathrm{~mm}^{3}\right) 3$ in the end plate configuration, and the temperature is $4.2 \mathrm{~K}$. In the upper panel, we plot linear amplitude versus phase. The data points (squares) form a perfect circle passing through the origin, indicating negligible leak vector. The frequency interval between each point is approximately $250 \mathrm{kHz}$ and the solid line is a fit to the data. In the lower panel, we plot both phase and linear amplitude (normalized) versus frequency. This resonance is perfectly symmetric due to the fact that the leak amplitude is $34.5 \mathrm{~dB}$ below the amplitude on resonance. The loaded $Q$-value of the cavity is 
19,000 and, thus, the resonance width $(2.34 \mathrm{MHz})$ is considerably less than the standing wave period, which is on the order of $100 \mathrm{MHz}$. The absqlute value of the phase returned by the $\mathrm{VR}$ is arbitrary, 36 which is why the phase on resonance is $64^{\circ}$ rather than $0^{\circ}$ (see Fig. 4a). In any subsequent experiment, we would null the phase on resonance, and interpret changes in the complex parameters of the signal returned to the VR according to the procedure described in section II-B.2. It should be noted that this data is about as good as one could expect were the cavity mounted on the bench top and the HG and HM connected directly to the cavity, i.e. the influence of the intervening waveguides has been completely eliminated.

Next, we consider the influence of the nearby TM111 mode on measurements made at the TE011 resonance frequency. Fig. 11 shows two such resonances obtained at liquid helium temperature (cavity A): the main part of the figure plots linear amplitude versus frequency; the inset shows the circles in the complex plane obtained for each of the resonances. The TM111 mode has been shifted $230 \mathrm{MHz}$ below the TE011 mode, which corresponds to almost 100 times the width of the TE011 mode $\left(\boldsymbol{\Gamma}_{T E 011}=2.42 \mathrm{MHz}\right)$ and about 40 times the width of the TM111 mode $\left(\boldsymbol{\Gamma}_{T M 111}=5.73 \mathrm{MHz}\right)$. The resonance amplitude of the TM111 mode is about $60 \%$ of the TE011 resonance amplitude. However, more importantly, the power of the TM111 signal (Obtained by extrapolation of a Lorentzian fit) is $44.5 \mathrm{~dB}$ below the power of the TE011 signal when the TE011 mode is at resonance. Thus, for all intents and purposes, we can rule out any interference between these modes. Even if there were a slight mixing, both modes have $\tilde{\mathbf{H}}$-fields perpendicular to the applied DC field $\left(\mathbf{B}_{\mathbf{o}}\right)$ for the end-plate configuration, and the TM111 mode has an $\tilde{\mathbf{H}}$-field node at the center of the cavity where the sample is usually placed in the quartzpillar configuration; this has been verified experimentally using electron paramagnetic resonance standards.37

$Q$-values as high as 24,900 have been obtained at liquid helium temperatures for the loaded axial cavities excited in TE011 modes. In general, higher $n$ (higher $f_{o}$ ) TE01 $n$ modes have reduced $Q$-values. In addition, the shorter wavelengths associated with the higher frequencies slightly increases the leak amplitude relative to the signal transmitted through the cavity. These facts, together with the diminished dynamic range of the spectrometer (see Table I) at higher frequencies, make it harder to observe TE01n $(n>1)$ resonances of comparable quality to the TE011 modes. Nevertheless, the data in Fig. 10 far exceeds the criteria discussed in section II-B for making successful cavity perturbation measurements. Consequently, we have been able to make reliable measurements at frequencies up to $130 \mathrm{GHz}$.

Above about $130 \mathrm{GHz}$, we have less confidence in the mode assignment of the resonances. However, by following the frequency dependence of data containing distinct features which also depend strongly on the polarization of the AC-fields within the cavity (see following section), we have been able to characterize and use axial cavity modes all the way up to $180 \mathrm{GHz}$. Fig. 12 shows several higher $n$ TE01 $n$ axial cavity modes, together with selective higher frequency resonances. Table II lists the frequencies, $Q$-values, leak amplitudes and dynamic ranges associated with these modes, as well as some parameters for the transverse cavity (all at $4.2 \mathrm{~K}$ ). These figures clearly demonstrate the potential of the system for cavity perturbation measurements. It should also be noted from Fig. 12 that many of the resonances were obtained using a single resonator (cavity A), which was one of the main objectives for this system from the very outset. 31 In the following section, we show real data obtained over the frequency range covered by this single cavity.

Finally, Fig. 13 illustrates the importance of using active temperature stabilization: the upper panel shows magnetic resonance data taken at the base temperature of the cryostat $(1.4 \mathrm{~K})$; the upper panel shows the same data obtained using active temperature stabilization at $1.8 \mathrm{~K}$. A clear drift in the unlocked temperature data is observed throughout the course the up and down sweeps of the magnetic field.

\section{B. Experimental examples}

By eliminating the influence of all components of the sample probe (aside from the cavity) on the signal returned to the VR, it is possible to record changes in the complex cavity parameters in real time, i.e. the vector (either amplitude and phase, or amplitude and frequency) recorded at the $\mathrm{VR}$ is directly related to the impedance of the cavity, $\mathbf{Z}_{\mathbf{C}}$. Here, once again, we see the power of the MVNA. Using a scalar detection scheme, it would be necessary to modulate the frequency in order to extract the complex cavity response.20 This would inevitably result in a much longer time for recording each data point. The MVNA essentially returns phase and amplitude information at the detection frequency of the VR, which is approximately $10 \mathrm{kHz} 1 \mathrm{~d}, 38$ This aspect of the instrument described in this paper makes it highly suited to measurements in high magnetic fields, which can be expensive to run for long periods.

A distinct advantage of conducting fixed frequency optical measurements, as a function of magnetic field, is that the spectral features which are under investigation may be expected to change with field, i.e. the magnetic field is the variable which is used to tune the electronic excitation spectrum of the material under investigation. If, for example, this induces a change from an insulating state, to a metallic state, then the optical response at frequencies comparable to the gap will reflect this change. A beautiful example of this can be seen from data obtained at the NHMFL, (Fig. 14) which shows the complex electrodynamic response of the organic superconductor (TMTSF) ${ }_{2} \mathrm{ClO}_{4}$, at low temperatures, as the magnetic field is swept to 30 tesla. 39 A rich behavior is observed; each of the more pronounced features may be attributed to modifications in the electronic configuration of the system which are known to occur in the field ranges 
from 5 to 10 tesla (Field-Induced-Spin-Density-Waves FISDW), and from 20 to 30 tesla (a phase line within the final FISDW phase). 40 Magneto-quantum-oscillations are also observed at high fields.

The data displayed in Fig. 14 were obtained using a phase lock while recording changes in amplitude $(A)$ and the resonance frequency $\left(f_{o}\right)$ of an axial cavity excited at $47.2 \mathrm{GHz}$. The sample was oriented with its $c^{*}$ axis parallel to the applied DC magnetic field, $\mathbf{B}_{\mathbf{o}}$, and currents were excited in the $a b$ plane of the sample. Each field sweep took less than 10 minutes. The quality of this data is, at worst, comparable to an equivalent DC measurement. From the combined information obtained from changes in dissipation $(\Delta A)$ and dispersion $\left(\Delta f_{o}\right)$ within the cavity, we can obtain valuable information about the electrodynamics of the byynd and free carrier systems in this fascinating material 39,40

The above example is quite different from a zero-field measurement where data are usually taken at many frequencies in order to investigate a particular spectral feature. Such experiments are generally plagued by poor dynamic range (signal-to-noise), especially in the millimeter and sub-millimeter spectral ranges. This is because identical coupling between the sample and the spectrometer cannot be guaranteed at each frequency, resulting in a large scatter of the data. This is unfortunate, since the cavity perturbation technique is inherently sensitive and, through the use of a suitable spectrometer $(e . g$. the MVNA), provides plenty of dynamic range at any given frequency. The instrument described in this paper is, instead, optimized at each frequency in order to detect minute changes in the optical conductivity of a sample as a function of magnetic field. These changes may subsequently be normalized to the zero-field conductivity using an appropriate instrument.

Whether to choose a phase lock or a frequency lock depends on the experiment. A frequency lock generally provides better stability because of the higher $Q$-value of the quartz frequency reference, as compared to the cavity. Fig. 15 shows an example of separate measurements using both techniques. The sample under investigation is the purple bronze $\eta-\mathrm{Mo}_{4} \mathrm{O}_{11} .41$ Fig. 15a shows variations in amplitude for both up and down sweeps of the magnetic field. There is a slight hysteresis, which is well documented for this material. However, the transmitted amplitude is considerably lower at high magnetic fields for the frequency locked measurement. This can be attributed to the cavity going well off resonance, as evidenced by a phase change of $25^{\circ}$ in the first 7 or 8 tesla (Fig 15b). Inspection of Fig. 4 clearly illustrates that a $25^{\circ}$ phase shift will lead to such a reduction in amplitude, even if there is no change in the dissipation within the cavity. Indeed, the ratio of the amplitudes obtained by each technique scales nicely with the cosine of the phase shift, as shown in the inset to Fig. 15b. Consequently, the frequency lock is inappropriate in this case, because of considerable mixing of the dissipative and dispersive responses of the sample in the phase and amplitude re- turned to the VR. Fig. 15c shows the frequency shift observed using the phase lock.

Fig. 16 shows Periodic Orbit Resonances (PORs - related to cyclotron resonance)42 observed through the inter-layer conductivity of the quasi-two-dimensional organic conductor $\alpha-(\mathrm{BEDT}-\mathrm{TTF})_{2} \mathrm{TlHg}(\mathrm{NCS})_{4} .43,44$ The frequencies, which are indicated above each trace in the figure, were obtained using a single axial cavity, and range all the way from $44 \mathrm{GHz}$ to $182 \mathrm{GHz}$. The sample was mounted in the end-plate configuration. Not all of the frequencies correspond to TE01n modes. However, from the shapes of the resonances ( $\sim$ Lorentzian peaks), we can be certain that the sample sits in the same electromagnetic environment for all of the modes, i.e. with the polarization of the oscillatory $\tilde{\mathbf{H}}$-field parallel to the conducting layers, resulting in the excitation of inter-layer currents (see Fig. 9d). This can be confirmed by studying the same sample in the TE011 quartz pillar configuration, as shown in Fig. 17, which is the conventional geometry for observing cyclotron resonance. Because of a high in-plane conductivity, it is the in-plane surface resistance of the samplethat governs the dissipation in the cavity (see Fig. 9c).27 45 This is the reason for the rather unconventional lineshape, i.e. an inflection rather than a symmetric dip or peak.

The data in Figs 16 and 17 are exceptional in their quality when compared to earlier attempts to measure cyclotron resonance in organic conductors 46 Furthermore, the resonance line shapes agree precisely with theory, thereby providing absolute confidence in the ability of the technique to discriminate between in-plane and interlayer transport phenomena in quasi-two-dimensional conductors. This has traditionally been problematic using conventional DC resistivity probes, because of uncertainties in the current paths within the samples. This opens up a huge range of possibilities for tackling issues concerning the role of electronic dimensionality in the physical properties of low-dimensional systems.

\section{SUMMARY}

We have described an instrument for conducting millimeter-wave cavity perturbation measurements over a continuously tunable frequency range $(40-200 \mathrm{GHz})$. The system is compatible with magnets both at Montana State University (up to 9 tesla) and at the National High Magnetic Field Laboratory (up to 33 tesla) in Tallahassee, FL. The utilization of a Millimeter-wave Vector Network Analyzer enables simultaneous measurements of the complex cavity parameters (resonance frequency and $Q$-value) at a rapid repetition rate $(\sim 10 \mathrm{kHz})$. Several experimental examples are presented which demonstrate the potential of this system for studying the magnetoelectrodynamics of low-dimensional conducting systems.

\section{ACKNOWLEDGEMENTS}

We are indebted to Norm Williams for technical assistance and to Prof. J. S. Brooks for the use of the MVNA 
at the NHMFL. This work was supported in part by the Office of Naval Research, and by NSF cooperative agreement No. 98-71922 with the state of Montana. Work carried out at the NHMFL was supported by a cooperative agreement between the State of Florida and the NSF under DMR-95-27035.

† email: hill@physics.montana.edu

$\ddagger$ email: abmm001@ibm.net

$\S$ email: email: gross@lkb.ens.fr

${ }^{1}$ P. A. Cox, Transition Metal Oxides (Clarendon, Oxford, 1995).

${ }^{2}$ T. Ishiguro and K. Yamaji, Organic Superconductors, in Springer Series in Solid State Sciences, $8 \mathbf{8}$ (SpringerVerlag, Berlin, 1990).

${ }^{3}$ See e.g. Physics and Applications of Quantum Wells and Superlattices Vol B170 of NATO ASI series, eds E. E. Mendez and K. von Klitzing (Plenum Press, 1987).

${ }^{4}$ F. J. Himpsel, J. E. Ortega, G. J. Mankey and R. F. Willis, Advances in Physics Vol. 47, No. 4, 511-597 (1998).

${ }^{5}$ J. G. Bednorz and K. A. Müller, Z. Phys. B 64, 189 (1986).

${ }^{6}$ Y. Moritomo, A. Asamitsu, H. Kuwahara and Y. Tokura, Nature (London) 380, 141 (1996).

${ }^{7}$ S. Hill, J. S. Brooks, S. Uji, T. Terashima, H. Aoki, Z. Fisk and J. Sarrao, Phys. Rev. B 58, 10778 (1998).

${ }^{8}$ S. Hill, P. S. Sandhu, C. Buhler, S. Uji, J. S. Brooks, L. Seger, M. Boonman, A. Wittlin, J. A. A. J. Perenboom, P. Goy, R. Kato, H. Sawa and S. Aonuma, in Millimeter and Submillimeter Waves III, Mohammed N. Afsar, Editor, Proc. SPIE 2842, pp 296-306 (1996).

${ }^{9}$ S. Hill, J. S. Brooks, J. S. Qualls, T. Burgin, B. Fravel, L. K. Montgomery, J. Sarrao and Z. Fisk, Physica B 246-247, 110 (1998).

${ }^{10}$ P. Goy, M. Gross, and J. M. Raimond, in Proc. 15th Int. Conf. in Infrared and Millimeter Waves, edited by R. J. Temkin Proc. SPIE 1514, 173-174 (1990).

${ }^{11}$ S. Hill, D. Phil thesis, University of Oxford, United Kingdom (1994).

12 M. E. J. Boonman, PhD thesis, University of Nijmegen, The Netherlands (1998).

13 This may be extended to $1 \mathrm{THz}$ through the association with Gunn oscillators.

${ }^{14}$ French Patent CNRS-ENS 1989, extended by AB Millimètre to Europe, Japan and the USA: P. Goy and M. Gross, US Patent Number 5119035 June 2, 1992, Millimeter and/or Submillimeter Network Vector Analyzer.

15 The numbers after MVNA 8-350 refer to the precise configuration of the MVNA. In this case it is the configuration with a single source and a single detector, and with a dual channel receiver, allowing vector detection of two microwave frequencies at the same time, or the vector detection of a signal and its derivative versus magnetic field.

${ }^{16}$ Examples of other high magnetic field laboratories having MVNAs: NHMFL, Tallahassee, FL, USA - Prof. J. S. Brooks and Prof. L-C. Brunel; Clarendon Laboratory,
University of Oxford, United Kingdom - Dr. John Singleton; University of Munich (FRG) - Prof. J. P. Kotthaus; High Field Magnet Laboratory, University of Nijmegen, The Netherlands - Prof. J. A. A. J. Perenboom; Institute for Materials Research (IMR), Tohoku University, Japan - Prof. M. Motokawa; The Institute for Physical and Chemical Research RIKEN, Japan - Prof. K. Katsumata; Research Institute for Advanced Science and Technology, University of Osaka, Japan - Prof. N. Toyota.

${ }^{17}$ Now available from Phase Metrix, 109 Bonaventura Drive, San Jose, CA 95134, USA (www.phasemetrix.com).

18 Available from Penn Engineering, 12750 Raymer Street, North Hollywood, CA 91605 (www.pennengineering.com).

19 The warm bore diameter of these magnets is $32 \mathrm{~mm}$. For measurements at pumped liquid helium temperatures, space is restricted to a $19 \mathrm{~mm}$ diameter bore.

${ }^{20}$ Available from AT Wall co., 55 Service Ave, Warwick, RI 02886, USA (www.atwall.com).

${ }^{21}$ The thermal conductivities at $77 \mathrm{~K}$ for SS, electrolytic $\mathrm{Cu}$ and $\mathrm{Ag}$ are $0.08,5.5$ and $3.1 \mathrm{~W} \mathrm{~cm}^{-1} \mathrm{~K}^{-1}$, respectively.

${ }^{22} \mathrm{SS}$ waveguide is manufactured with a $0.51 \mathrm{~mm}$ wall, as opposed to $1.02 \mathrm{~mm}$ for $\mathrm{Cu}$ and $\mathrm{Ag}$.

23 This is due to the high electrical resistivity of SS compared to $\mathrm{Ag}$ and $\mathrm{Cu}$. The electrical resistivities at $300 \mathrm{~K}$ for SS, electrolytic $\mathrm{Cu}$ and $\mathrm{Ag}$ are $72,1.67$ and $1.59 \mathrm{~m} \Omega \mathrm{cm}$, respectively.

24 The reason we use both $\mathrm{Cu}$ and $\mathrm{Ag}$ in the probe simply has to do with availability at the time of construction. Thus, at the lower end of the probe, one of the guides is $\mathrm{Cu}$ and the other is Ag.

25 The gold plating was performed by Custom Microwave Inc., 940 Boston Avenue, Longmont, CO 80501, USA (www.custommicrowave.com).

26 These parts were fabricated using a Computer Aided Machine at MSU.

27 O. Klein, S. Donovan, M. Dressel and G. Grüner, Int. J. of Infrared and Millimeter Waves, 14, 2423 (1993).

${ }^{28}$ S. Donovan, O. Klein, M. Dressel, K. Holczer and G. Grüner, Int. J. of Infrared and Millimeter Waves, 14, 2423 (1993).

29 M. Dressel, O. Klein, S. Donovan, G. Grüner, Int. J. of Infrared and Millimeter Waves, 14, 2423 (1993).

${ }^{30}$ More precisely, $1 \mathrm{~m}$ of cable compensates for $1.18 \mathrm{~m}$ in air (vacuum). However, in waveguides close to cut-off, this conversion factor may not be reliable.

${ }^{31}$ Although our goal is to be able to measure at many different frequencies with a single cavity, different cavities are needed for different experiments, e.g. a long cavity will provide many closely spaced modes within a narrow frequency range, whereas a short cavity would spread these modes out over a broad frequency range.

${ }^{32}$ C. P. Poole, Electron Spin Resonance (Interscience, New York 1975).

33 This will shortly increase to $45 \mathrm{~T}$ when the hybrid magnet comes on line.

34 J. S. Brooks, J. E. Crow and W. G. Moulton, J. Phys. Chem. Solids 59, No 4, 569 (1998).

35 S. Hill, S. Uji, P. S. Sandhu, M. Chaparalla, J. S. Brooks and L. Seger, Synth. Met. 86, 1955 (1997).

${ }^{36}$ In addition to the phase shift across the cavity, the phase 
returned to the VR includes an additional phase shift due to the path difference between the signals reaching the HM from $S_{1}$ and $S_{2}$.

${ }^{37} \mathrm{~S}$. Hill et al., to be published elsewhere.

${ }^{38}$ Although the VR detects at $10 \mathrm{kHz}$, it is common to average this signal over about 50 cycles, resulting in a detection frequency of about $200 \mathrm{~Hz}$.

${ }^{39}$ S. Hill; J.S. Brooks; J.S. Qualls; B.W. Fravel; L.K. Montgomery, accepted for publication in Synth. Met. (1998).

40 S. K. McKernan, S. T. Hannahs, U. M. Scheven, G. M. Danner and P. M. Chaikin, Phys. Rev. Lett. 75, 1630 (1995).

${ }^{41}$ S. Hill, S. Valfells, S. Uji, J. S. Brooks, G. J. Athas, P. S. Sandhu, J. Sarrao, Z. Fisk, J. Goettee, H. Aoki and T. Terashima, Phys. Rev. B 55, 2018 (1997).

${ }^{42}$ Stephen Hill, Phys. Rev. B 55, 4931 (1997).

43 Stephen Hill, M. Mola, J. S. Brooks, M. Tokumoto, N. Kinoshita, T. Kinoshita and Y. Tanaka, to be published in Proc. "Physical Phenomena in High Magnetic Fields III" (PPHMF-III), eds Z. Fisk, L. P. Gor'kov and J. R. Schri- effer (World Scientific, Singapore 1999).

${ }^{44} \mathrm{~S}$. Hill et al., in preparation.

${ }^{45}$ S. Hill, P. S. Sandhu, M. Boonman, J. A. A. J. Perenboom, A. Wittlin, S. Uji, J. S. Brooks, R. Kato, H. Sawa and S. Aonuma, Phys. Rev. B 54, 13536 (1996).

${ }^{46}$ See e.g: J. Singleton, F. L. Pratt, M. Doporto, T. J. B. M. Janssen, M. Kurmoo, J. A. A. J. Perenboom, W. Hayes and P. Day, Phys. Rev. Lett. 68, 2500 (1992); S. Hill, A. Wittlin, J. van-Bentum, J. Singleton, W. Hayes, J. A. A. J. Perenboom, M. Kurmoo and P. Day, Synth. Met. 70, 821 (1995); S. V. Demishev, A. V. Semeno, N. E. Sluchanko, N. A. Samarin, I. B. Voskoboinikov, V. V. Glushkov, J. Singleton, S. J. Blundell, S. O. Hill, W. Hayes, M. V. Kartsovnik, A. E. Kovalev, M. Kurmoo, P. Day and N. D. Kushch, Phys. Rev. B 53, 12794 (1996); A. Polisskii, J. Singleton, P. Goy, W. Hayes, M. Kurmoo and P. Day, J. Phys.: Cond. Mat. 8, L195 (1996); and A. Ardavan, J. M. Schrama, S. J. Blundell, J. Singleton, W. Hayes, M. Kurmoo, P. Day and P. Goy, Phys. Rev. Lett. 81, 713 (1998). 


\begin{tabular}{|c|c|c|}
\hline $\begin{array}{c}\text { Band, } \\
\text { harmonic }\end{array}$ & $\begin{array}{c}\text { Frequency } \\
\text { (GHz) }\end{array}$ & $\begin{array}{c}\text { Dynamic range - } \\
\text { analyzer (dB) }\end{array}$ \\
\hline $\mathrm{V}, \mathrm{N}=3$ & 48 & $>128$ \\
\hline $\mathrm{V}, \mathrm{N}=4$ & 60 & $>128$ \\
\hline $\mathrm{V}, \mathrm{N}=4$ & 70 & 126.4 \\
\hline $\mathrm{W}, \mathrm{N}=5$ & 79 & 105.7 \\
\hline $\mathrm{W}, \mathrm{N}=5$ & 88 & 103 \\
\hline $\mathrm{W}, \mathrm{N}=6$ & 99 & 94 \\
\hline $\mathrm{W}, \mathrm{N}=6$ & 108 & 83.8 \\
\hline $\mathrm{D}, \mathrm{N}=8$ & 119 & 83 \\
\hline $\mathrm{D}, \mathrm{N}=9$ & 135 & 76.8 \\
\hline $\mathrm{D}, \mathrm{N}=10$ & 157 & 65.3 \\
\hline $\mathrm{D}, \mathrm{N}=12$ & 186 & 57.8 \\
\hline
\end{tabular}

TABLE I. The dynamic ranges achieved using the MVNA-8-350 at MSU at various frequencies in each microwave band.

\begin{tabular}{|c|l|r|r|r|r|}
\hline Band/harmonic & Cavity/mode & $\boldsymbol{f}_{\mathbf{0}}(\mathbf{G H z})$ & \multicolumn{1}{c|}{$\boldsymbol{Q}$} & $\mathbf{S} / \mathbf{N}(\mathbf{d B})$ & $\mathbf{A}\left(\boldsymbol{f}_{\mathbf{0}}\right)-\mathbf{A}_{\boldsymbol{l}}(\mathbf{d B})$ \\
\hline $\mathrm{V}-3$ & $\mathrm{~A} 1-\mathrm{TE} 011$ & 44.450 & 19,000 & 76.0 & 34.5 \\
\hline $\mathrm{V}-3$ & $\mathrm{~A} 1-\mathrm{TM} 11$ & 44.219 & 7,700 & 74.6 & 19.0 \\
\hline $\mathrm{V}-3$ & $\mathrm{~B} 1-\mathrm{TE} 011$ & 44.414 & 24,900 & 81.4 & 26.0 \\
\hline $\mathrm{V}-3$ & $\mathrm{~B} 1-\mathrm{TM} 111$ & 44.265 & 7,400 & 80.3 & 13.8 \\
\hline $\mathrm{V}-4$ & $\mathrm{~A} 1-\mathrm{TE} 012$ & 58.754 & 13,900 & 85.4 & 27.0 \\
\hline $\mathrm{V}-4$ & $\mathrm{~A} 1-\mathrm{TE} 212$ & 53.951 & 10,400 & 87.8 & 40.4 \\
\hline $\mathrm{V}-4$ & $\mathrm{~A} 1-\mathrm{TE} 12$ & 61.247 & 10,000 & 90.4 & 24.5 \\
\hline $\mathrm{V}-4$ & $\mathrm{~A} 1-\mathrm{TE} 412$ & 68.906 & 4,800 & 92.4 & 20.8 \\
\hline $\mathrm{W}-5$ & $\mathrm{~A} 2-\mathrm{TE} 213$ & 73.924 & 8,800 & 84.5 & 25.0 \\
\hline $\mathrm{W}-5$ & $\mathrm{~A} 2-\mathrm{TE} 013$ & 78.044 & 7,900 & 89.0 & 23.0 \\
\hline $\mathrm{W}-5$ & $\mathrm{~A} 2$ & 88.678 & 23,000 & 90.0 & 22.0 \\
\hline $\mathrm{W}-6$ & $\mathrm{~A} 2-\mathrm{TE} 014$ & 98.715 & 5,300 & 83.0 & 16.5 \\
\hline $\mathrm{W}-6$ & $\mathrm{~A} 2$ & 103.532 & 4,350 & 84.5 & 27.0 \\
\hline $\mathrm{D}-8$ & $\mathrm{C} 3-\mathrm{TE} 014$ & 127.233 & 13,600 & 45.8 & 16.5 \\
\hline $\mathrm{D}-9$ & $\mathrm{C} 3$ & 145.227 & 7,900 & 48.5 & 17.5 \\
\hline $\mathrm{D}-10$ & $\mathrm{C} 3$ & 155.815 & 5,800 & 41.2 & 21.0 \\
\hline $\mathrm{D}-12$ & $\mathrm{C} 3$ & 187.686 & 5,150 & 31.0 & 4.0 \\
\hline $\mathrm{V}-4$ & $\mathrm{~T}-\mathrm{TE} 012$ & 56.110 & 15,500 & 81.9 & 18.0 \\
\hline $\mathrm{V}-4$ & $\mathrm{~T}-\mathrm{TE} 013$ & 65.078 & 14,000 & 74.6 & 13.0 \\
\hline
\end{tabular}

TABLE II. Resonance parameters for different modes obtained in various different cavities. The first column lists the frequency band and the MVNA harmonic. The second column lists the cavity (letter) and end plate used (number), as well as the mode (if known); cavities $\mathrm{A}, \mathrm{B}$ and $\mathrm{C}$ have dimensions (length $\times$ diameter) $6.7 \mathrm{~mm} \times 9.52 \mathrm{~mm}, 8.73 \mathrm{~mm} \times 9 \mathrm{~mm}$, and $5.54 \mathrm{~mm} \times 5.64 \mathrm{~mm}$ respectively, cavity $\mathrm{T}$ is the transverse cavity $(10.16 \mathrm{~mm} \times 7.75 \mathrm{~mm})$, and end plates 1,2 and 3 have coupling hole diameters of $1.32 \mathrm{~mm}, 0.84 \mathrm{~mm}$ and $0.62 \mathrm{~mm}$, respectively. The next three columns list the resonance frequencies $\left(f_{o}\right), Q$-values and dynamic ranges (signal-to-noise) for each mode. The final column lists the contrast in dB between the transmitted amplitude on resonance $\left[A\left(f_{o}\right)\right]$ and the leak amplitude $\left(A_{l}\right)$. The dynamic range is somewhat lower at $45 \mathrm{GHz}$ than in the main part of the $\mathrm{V}$ - and W-bands due to the fact that the V-band wave-guide is so close to cut off at these low frequencies. 


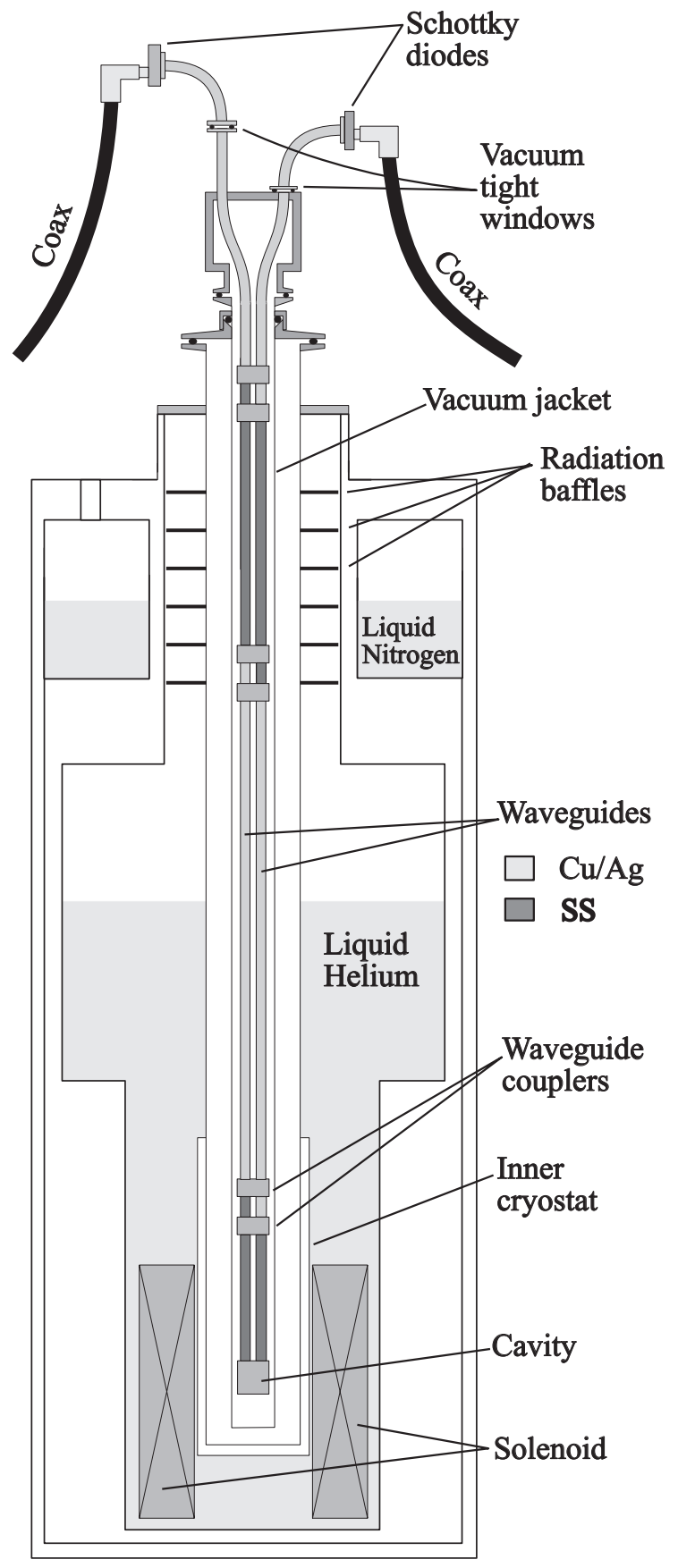

FIG. 1. Schematic of the sample probe and superconducting magnet system at MSU (not to scale).

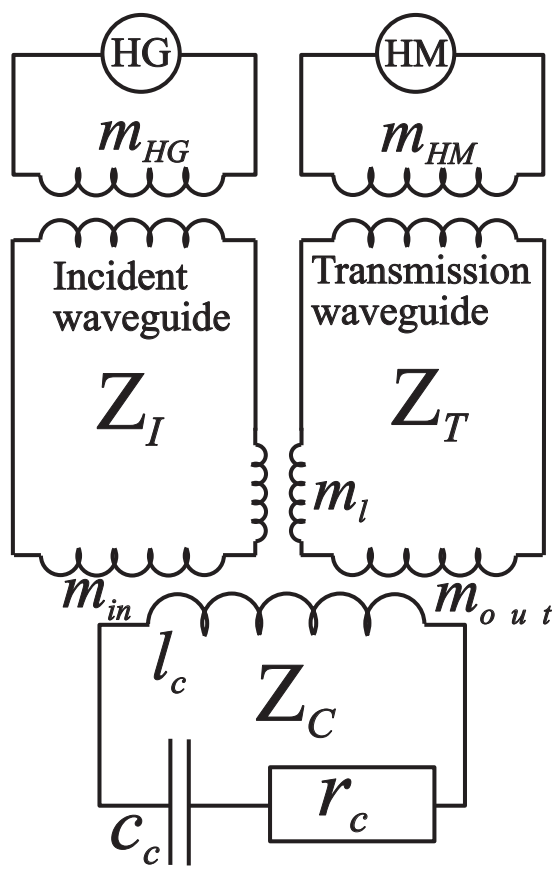

FIG. 2. An equivalent LRC circuit representation of the key microwave components of the sample probe. The coupling between $\mathrm{HG}(\mathrm{HM})$ and the incidence (transmission) waveguide is modeled as a coupling mutual inductance $m_{H G}$ $\left(m_{H M}\right)$. The cavity is coupled to each of the waveguides through coupling mutual input $\left(m_{i n}\right)$ and output $\left(m_{\text {out }}\right)$ inductances. A leak signal between the incidence and transmission waveguides, in parallel with the cavity, is modeled as a direct coupling mutual inductance, $m_{l}$, between the two waveguides.

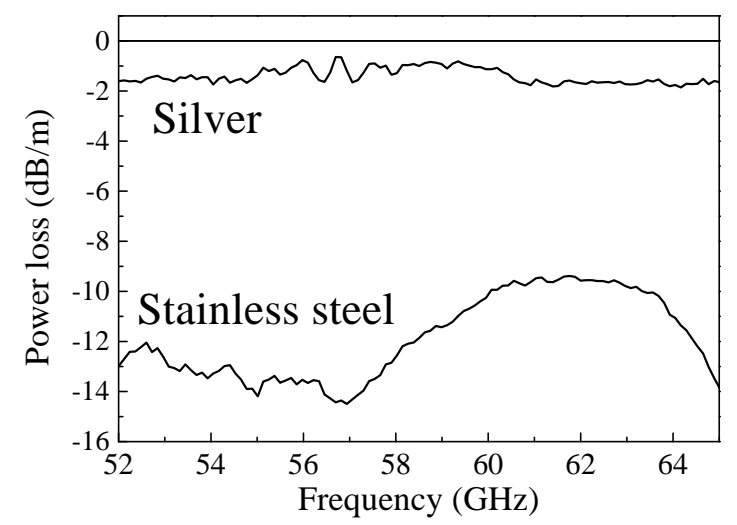

FIG. 3. A comparison between the insertion losses (in $\mathrm{dB} / \mathrm{m}$ ) for stainless steel and Silver waveguide sections in $\mathrm{V}$-band at room temperature. 


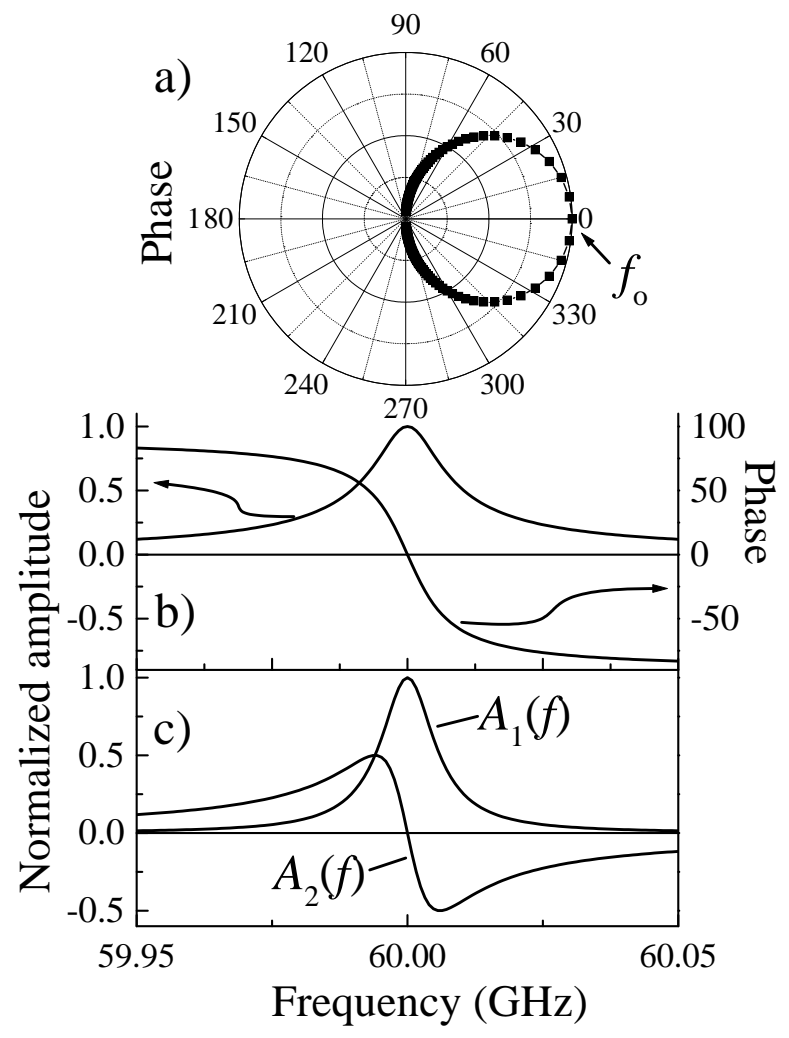

FIG. 4. Simulation of the complex parameters of a wave transmitted through a cavity which is resonant at $60 \mathrm{GHz}$, and has a $Q$ of 5000 ; a) a plot of linear amplitude versus phase in the complex plane; b) a plot of linear amplitude (normalized) and phase versus frequency; and c) a plot of the real $\left(A_{1}\right)$ and imaginary $\left(A_{2}\right)$ linear amplitudes (normalized) versus frequency.

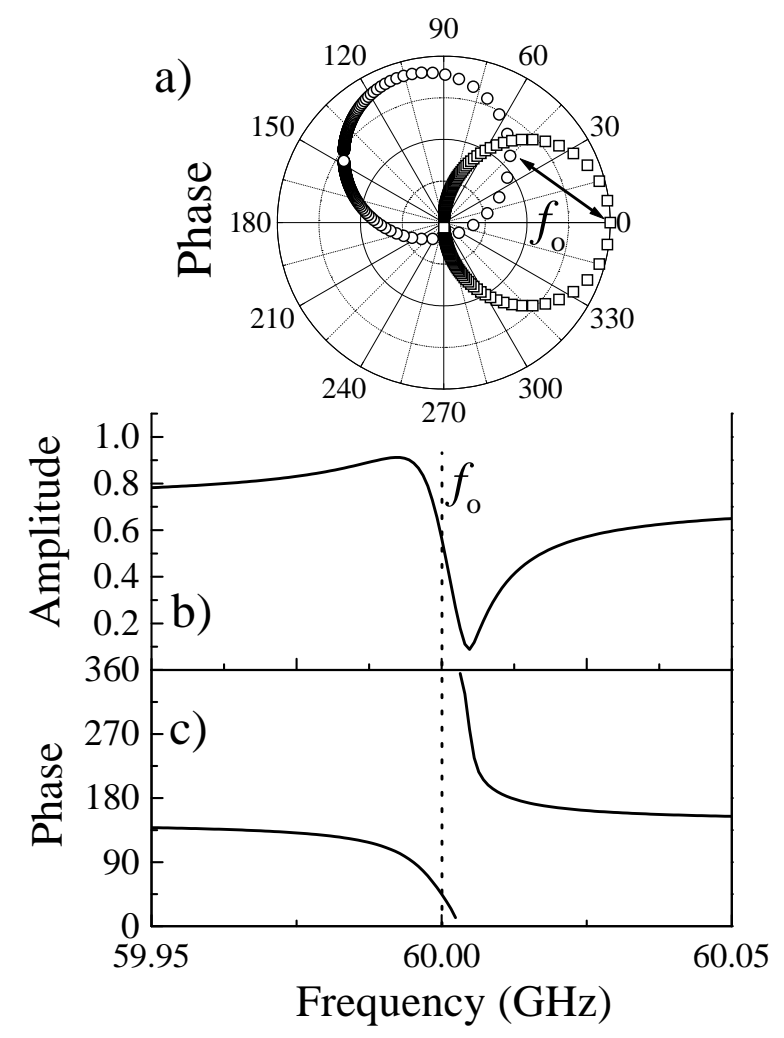

FIG. 5. A demonstration of the effect of a leak wave on the ideal resonance in Fig. 4. The complex leak vector translates the resonance in the complex plane, as shown by the arrow in a). The result is that the resonance frequency, $f_{o}$, no longer lies along the real axis $(\phi=0)$. Thus, $f_{o}$ no longer corresponds to the maximum amplitude, resulting in the distorted Lorentzian lineshape shown in b) and c). 


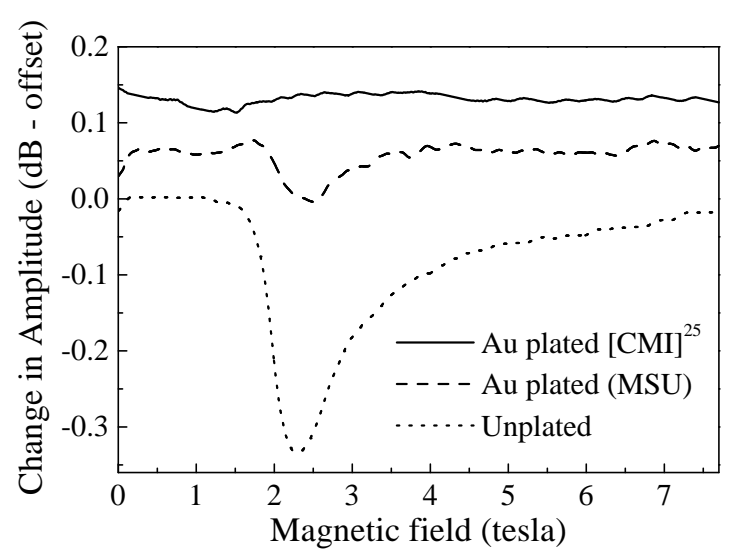

FIG. 6. A comparison between the background (empty $\mathrm{Cu}$ cavity) response of the sample probe using Gold plated and unplated stainless steel sections at the lower end of the probe; these data were obtained at $76.7 \mathrm{GHz}$ and $4.2 \mathrm{~K}$. The broad dip is attributed to an EPR absorption due to small quantities of $\mathrm{Fe}^{3+}$ (rust) at the surface of the SS. Professional gold plating (solid line) clearly eliminates this contamination, which is evident both in the unplated data (short dash) and the data obtained after our efforts to plate the SS at MSU (long dash).

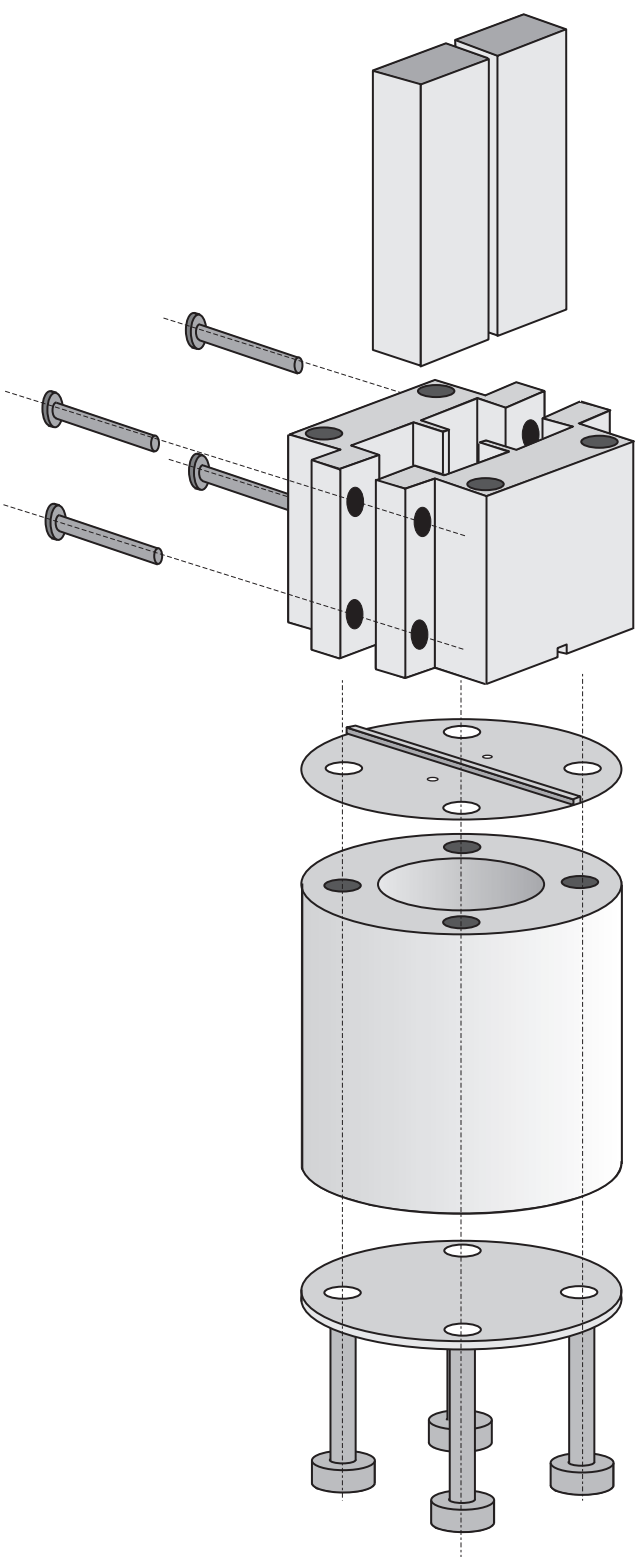

FIG. 7. Schematic of the axial cavity construction. See text for detailed description. 


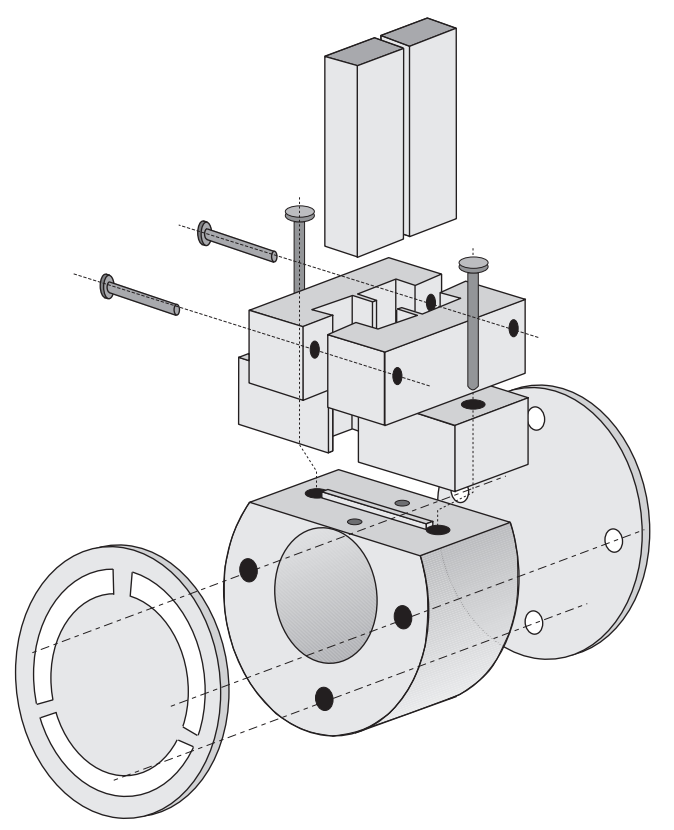

FIG. 8. Schematic of the transverse cavity construction. See text for detailed description. a)


FIG. 9. a) The end plate cavity perturbation configuration, showing the position of the sample within a TE011 cavity and the radial $\tilde{H}$-field at the position of the sample. b) The quartz pillar configuration, showing the sample at the heart of a TE011 cavity atop a quartz pillar, and the axial $\tilde{H}$-field at the position of the sample. c) Excitation of in-plane currents at the edges of a Q2D conductor. d) Excitation of inter-layer currents within the bulk of a Q2D conductor. In both c) and d), the low conductivity direction is parallel to the normal to the disc shaped sample. e) Sample rotation for the quartz pillar configuration in the transverse cavity - note that the orientation of the axial AC $\tilde{H}$-field does not change relative to the sample upon rotation. 


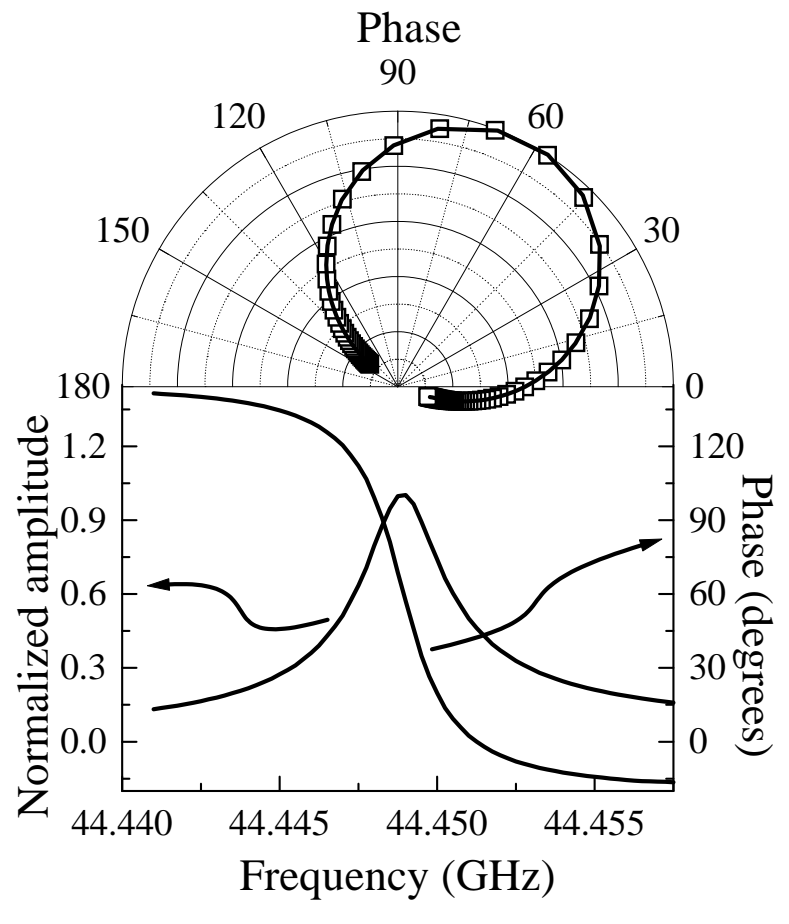

FIG. 10. A real TE011 resonance (raw data at $4.2 \mathrm{~K}$ ) for a loaded axial cavity (cavity A). The upper panel shows the resonance circle in the complex plane - the squares are raw data and the solid line is a fit. The lower panel shows the linear amplitude and phase variation as a function of frequency.

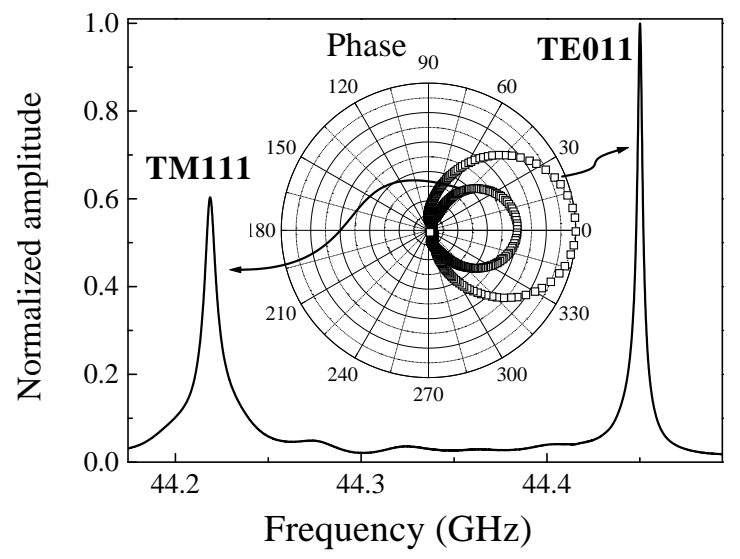

FIG. 11. Lifting the degeneracy of the TE011 and TM111 modes $(\mathrm{T}=4.2 \mathrm{~K})$ in cavity $\mathrm{A}$. The main part of the figure shows the two resonances (linear scale) separated by 230 $\mathrm{MHz}$. The inset shows the circles in the complex plane corresponding to the two resonances.
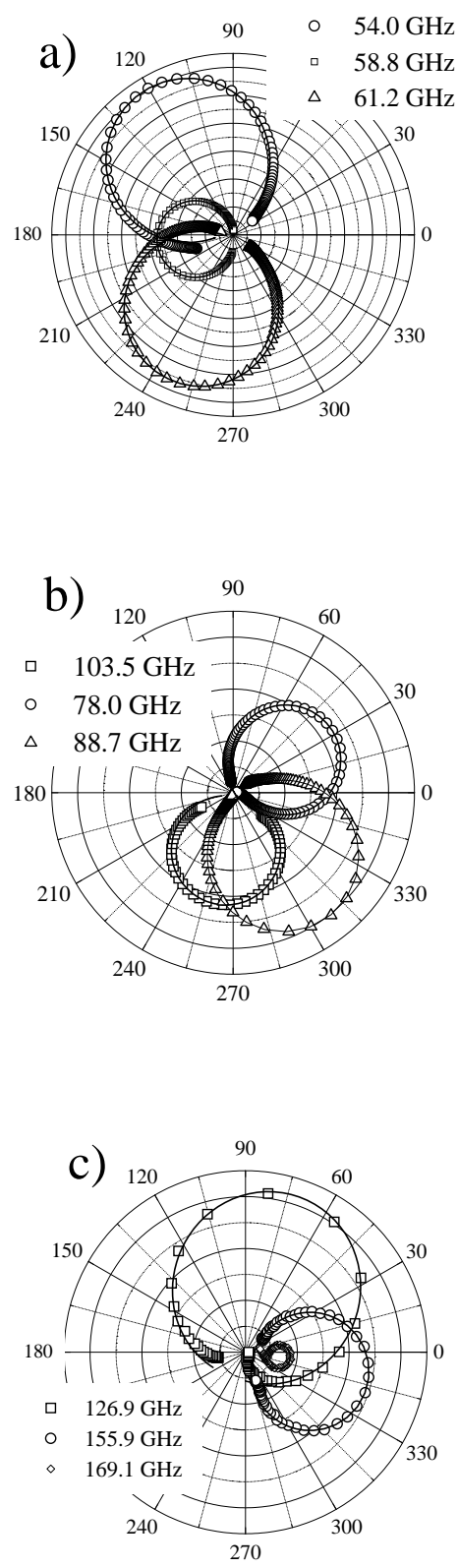

FIG. 12. Various cavity resonances seen as circles in the complex plane, obtained in a) V-band, b) W-band and c) D-band. The points are raw $4.2 \mathrm{~K}$ data and the solid lines represent Lorentzian fits to the data. See table 2 for more details about each of the resonances. 


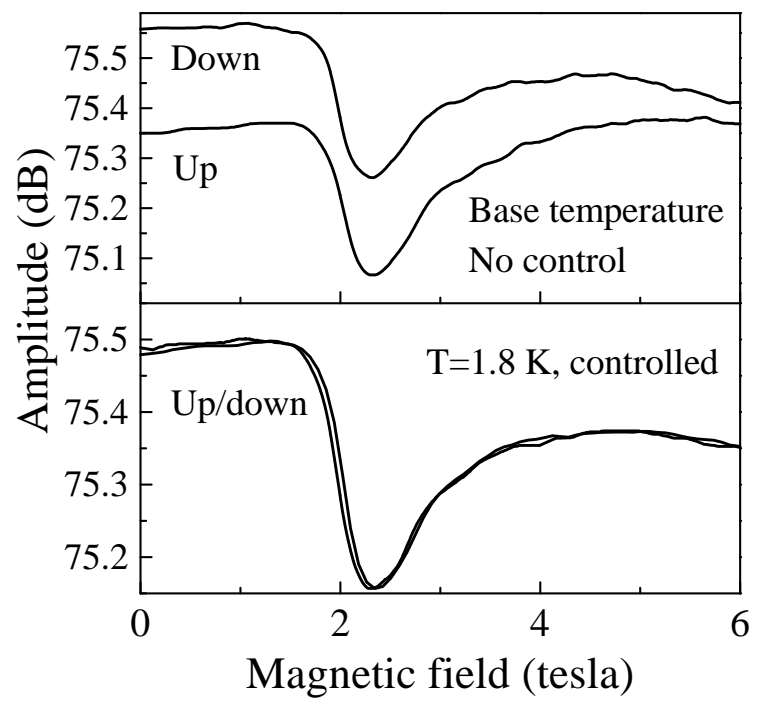

FIG. 13. A comparison between magnetic field sweeps with the temperature unlocked (upper panel) and actively controlled (lower panel). The broad dip corresponds to the $\mathrm{Fe}^{3+}$ resonance seen in Fig. 6. Note: this data was obtained prior to $\mathrm{Au}$ plating the lower SS section of the probe.

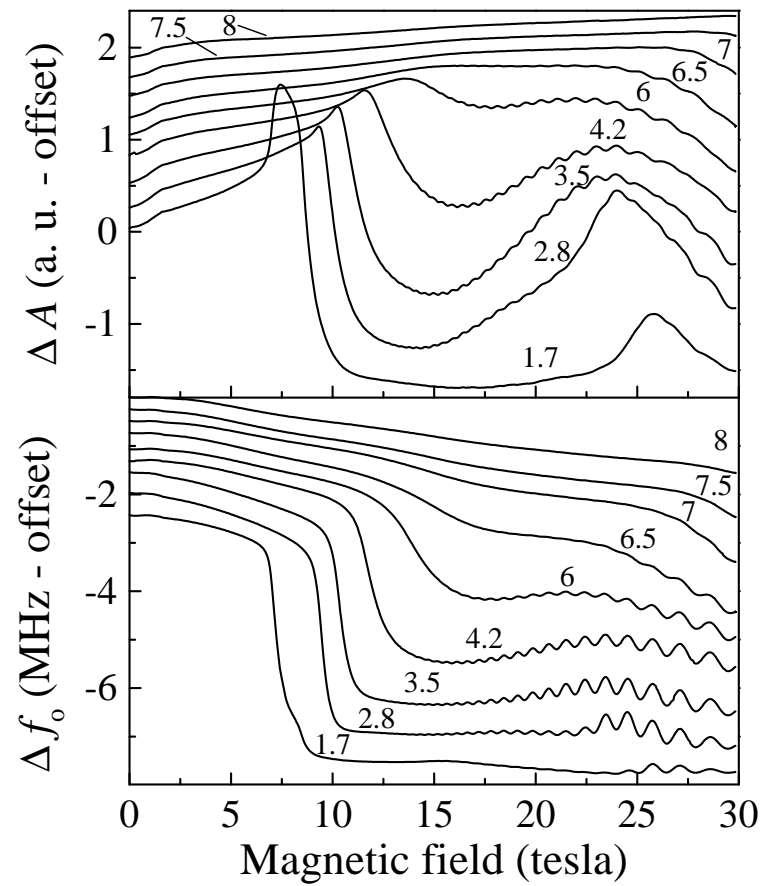

FIG. 14. Dissipative $(\Delta A)$ and reactive $\left(\Delta f_{o}\right)$ responses of (TMTSF $)_{2} \mathrm{ClO}_{4}$ in a magnetic field. The data were obtained in an axial TE011 cavity excited at $47.2 \mathrm{GHz}$. The temperatures are indicated in kelvin in the figure, and the traces have been offset for the sake of clarity. 


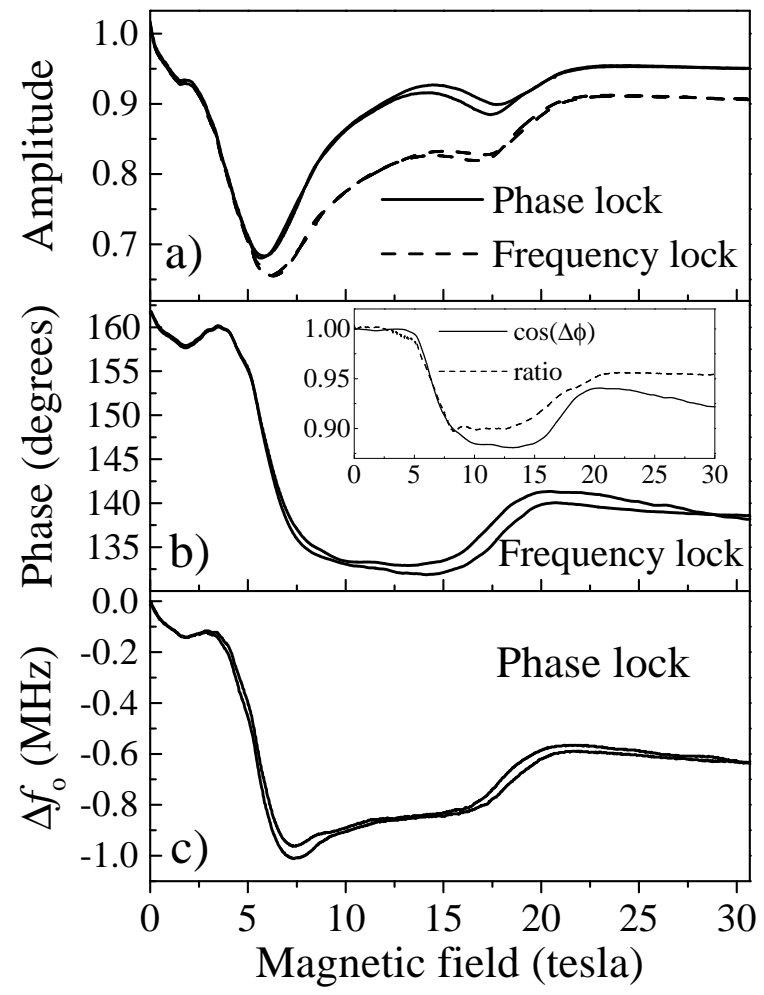

FIG. 15. a) A comparison between the amplitude variation versus magnetic field obtained using frequency and phase locking techniques. The sample is the purple bronze $\eta-\mathrm{Mo}_{4} \mathrm{O}_{11}$, and the observed changes are due to the excitation of inter-layer currents in this quasi-two-dimensional conductor. b) Shows the phase variation during the frequency locked measurement (absolute microwave frequency fixed at $44.272 \mathrm{GHz}$ ), while c) shows the frequency variation during the phase locked measurement (absolute microwave phase across the sample probe locked). The inset to b) compares the ratio of the amplitudes obtained for the two techniques in a), with the cosine of the phase change in the main part of b). The good correspondence between these quantities confirms that the difference between the traces in a) can be attributed to the large phase shift during the frequency locked measurement. Thus, a phase lock should be applied in this situation.

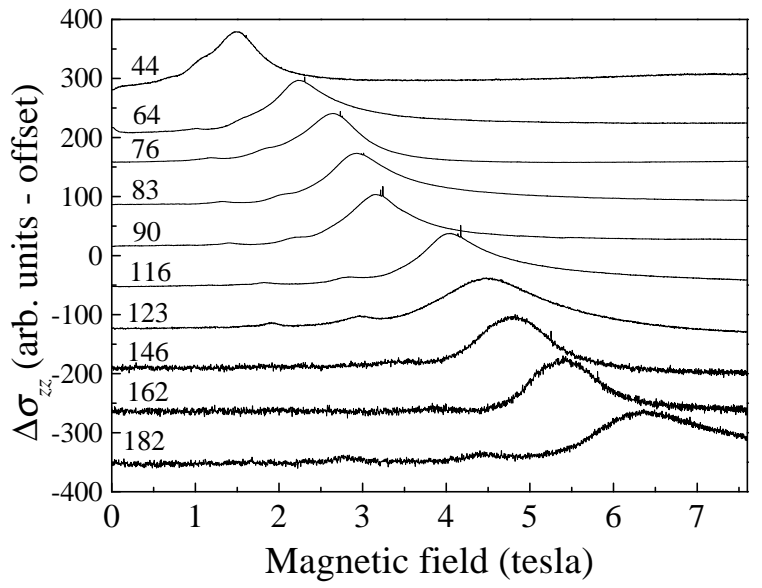

FIG. 16. Frequency dependence of the Periodic Orbit Resonances observed through the inter-layer conductivity $\left(\sigma_{z z}\right)$ in $\alpha-(\mathrm{BEDT}-\mathrm{TTF})_{2} \mathrm{TlHg}(\mathrm{NCS})_{4}$, when mounted in a TE011 axial cavity in the end plate configuration; the temperature is $1.8 \mathrm{~K}$. The data appear noisier at higher frequencies due to the reduced dynamic range of the spectrometer and due to the reduced sensitivity (lower $Q$-values) of the higher order cavity modes. The resonances have been scaled so as to appear the same strength, and have been offset for the sake of clarity. The sharp spikes observed slightly above the main peak positions are Electron Paramagnetic Resonance signals due to a DPPH sample placed in the cavity.

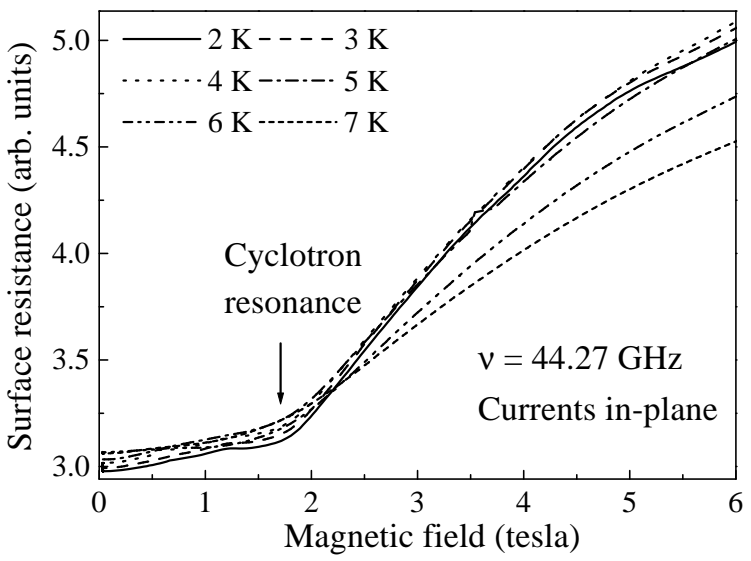

FIG. 17. Conventional cyclotron resonances seen through the in-plane surface resistance for the same sample as in Fig. 16, this time mounted in a TE011 axial cavity in the quartz pillar configuration; the frequency is $44.27 \mathrm{GHz}$ and the temperatures are indicated in the figure. The resonances are observed as sharp inflections which weaken as the temperature increases. 